\title{
The flow topology transition of liquid-liquid Taylor flows in square microchannels
}

\author{
Thorben Helmers ${ }^{1} \cdot$ Philip Kemper $^{2} \cdot$ Jorg Thöming ${ }^{2}$. Ulrich Mießner ${ }^{1}[$
}

Received: 5 January 2021 / Revised: 1 October 2021 / Accepted: 20 November 2021 / Published online: 10 December 2021

(C) The Author(s) 2021

\begin{abstract}
This work investigates the change of the flow topology of Taylor flow and qualitatively relates it to the excess velocity. Ensemble-averaged 3D2C- $\mu$ PIV measurements simultaneously resolve the flow field inside and outside the droplets of a liquid-liquid Taylor flow that moves through a rectangular horizontal microchannel. While maintaining a constant Capillary number $\mathrm{Ca}=0.005$, the Reynolds number $(0.52 \leq \mathrm{Re} \leq 2.14)$, the viscosity ratio $(0.24 \leq \lambda \leq 2.67)$ and surfactant concentrations of sodium dodecyl sulfate ( $0-3 \mathrm{CMC}$ ) are varied. We experimentally identified the product of the Reynolds number Re and the viscosity ratio $\lambda$ to indicate the momentum transport from the continuous phase (slugs) into the droplets (plugs). The position and size of the droplet's main vortex core as well as the flow topology in the cross section of this vortex core changed with increased momentum transfer. Further, we found that the relative velocity of the Taylor droplet correlates negatively with the evoked topology change. A correlation is proposed to describe the effect quantitatively.
\end{abstract}

\section{Graphical abstract}

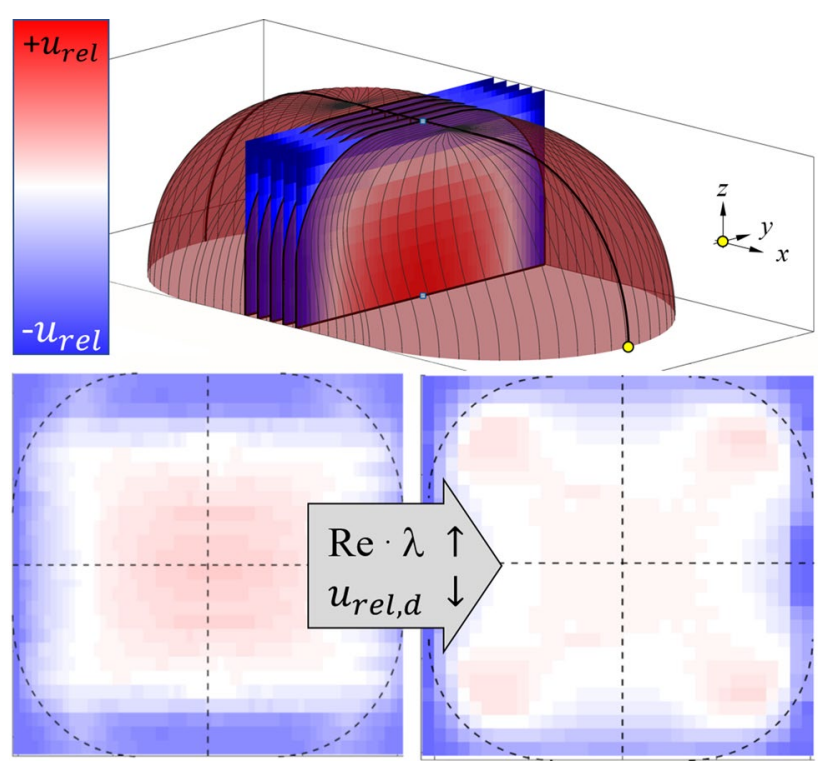

\section{List of symbols}

\section{Abbreviations}

2D2C Two-dimensional, two velocity components

3D2C Three-dimensional, two velocity components

Ulrich Mießner

miessner@uvt.uni-bremen.de

Extended author information available on the last page of the article
AOT Dioctyl sulfosuccinate sodium

CCD Charge-coupled device sensor

CMC Critical micelle concentration

$\mathrm{CV}$ Coefficient of variation

DMSO Dimethyl sulfoxide

DOC Depth of correlation

DOF Depth of field 


\begin{tabular}{|c|c|c|c|}
\hline IWS & Interrogation windows size & \multicolumn{2}{|c|}{ Superscripts } \\
\hline NA & Numerical aperture & $*$ & Dimensionless \\
\hline PDMS & Polydimethylsiloxane & · & Temporal derivative \\
\hline PIV & Particle image velocimetry & \multirow{2}{*}{\multicolumn{2}{|c|}{ Subscripts }} \\
\hline PTV & Particle tracking velocimetry & & \\
\hline px & Pixel & 0 & Superficial \\
\hline RI & Refractive index & c & Continuous phase \\
\hline RIM & Refractive index matching & $\mathrm{ch}$ & Channel \\
\hline SDS & Sodium dodecyl sulfate & corr & Corrected value \\
\hline & & d & Disperse/droplet phase \\
\hline \multicolumn{2}{|c|}{ Dimensionless quantities } & ex & Excess \\
\hline Bo & Bond number $\frac{\Delta \rho g H^{2}}{\sigma}$ & $\mathrm{g}$ & Gutter \\
\hline $\mathrm{Ca}$ & Capillary number $\frac{\sigma}{u_{0} \eta_{\mathrm{c}}}$ & $\max$ & Maximum value \\
\hline $\mathrm{La}$ & Laplace number $\frac{H \rho_{\mathrm{c} \sigma}^{\sigma}}{\eta^{2}}=1 / \mathrm{Oh}^{2}$ & rec & Recording \\
\hline $\mathrm{Oh}$ & Ohnesorge number $\frac{\eta_{\mathrm{c}}}{\sqrt{\eta_{\mathrm{c}}}}=\sqrt{\mathrm{Ca} / \mathrm{Re}}$ & rel & Relative \\
\hline $\mathrm{Re}$ & Revnolds number $\frac{\rho_{c} u_{0} H}{\sqrt{H \rho_{c} \sigma}}$ & shift & Shifted \\
\hline & Reynolds number $\frac{\eta_{\mathrm{c}}}{\eta_{\mathrm{c}}}$ & vort & Vortex \\
\hline
\end{tabular}

\section{Greek symbols}

$\beta \quad$ Dimensionless friction factor [-]

$\dot{\gamma} \quad$ Shear rate $\left[\mathrm{s}^{-1}\right]$

$\eta \quad$ Dynamic viscosity [mPas]

$\Lambda \quad$ Light wavelength $[\mu \mathrm{m}]$

$\lambda \quad$ Viscosity ratio $\frac{\eta_{d}}{\eta_{c}}$

$\Omega \quad$ Specific flow resistance [ $\left[\mathrm{Ns} \mathrm{m}^{-5}\right.$ ]

$\phi \quad$ Model parameter [-]

$\rho \quad$ Density $\left[\mathrm{kg} \mathrm{m}^{-3}\right]$

$\sigma \quad$ Interfacial tension $\left[\mathrm{N} \mathrm{m}^{-1}\right]$

$\theta \quad$ Opening angle of objective $\left[{ }^{\circ}\right]$

$\varepsilon \quad$ Minimal detectable area $\left[\mu \mathrm{m}^{2}\right]$

$\xi \quad$ Mass fraction [-]

\section{Roman symbols}

$\begin{array}{ll}A & \text { Area }\left[\mu \mathrm{m}^{2}\right] \\ d & \text { Diameter }[\mu \mathrm{m}] \\ f & \text { Frequency }\left[\mathrm{s}^{-1}\right] \\ g & \text { Gravitational acceleration }\left[\mathrm{m} \mathrm{s}^{-2}\right] \\ H & \text { Height }[\mu \mathrm{m}] \\ l & \text { Length }[\mu \mathrm{m}] \\ P & \text { Shape factor } \\ p & \text { Probability value of F test on the regression coef- } \\ & \text { ficient }[-] \\ Q & \text { Volumetric flow rate }\left[\mu 1 \mathrm{~s}^{-1}\right] \\ R & \text { Radius }[\mu \mathrm{m}] \\ r & \text { Pearson correlation coefficient }[-] \\ u & \text { Velocity in the } x \text {-direction }\left[\mathrm{m} \mathrm{s}^{-1}\right] \\ v & \text { Velocity in the } y \text {-direction }\left[\mathrm{m} \mathrm{s}^{-1}\right] \\ W & \text { Width }[\mu \mathrm{m}] \\ w & \text { Velocity in the } z \text {-direction }\left[\mathrm{m} \mathrm{s}^{-1}\right] \\ x & x \text {-direction }[\mu \mathrm{m}] \\ y & y \text {-direction }[\mu \mathrm{m}] \\ z & z \text {-direction/position of focal } \mathrm{plane}^{-1}\end{array}$

\section{Introduction}

The versatile possibilities of microfluidic applications have been recognized in the beginning millennium (Ehrfeld et al. 2004; Seeberger and Blume 2007; Angeli and Gavriilidis 2008; Dietrich 2009; Huebner et al. 2008). Herein, microscopic multiphase flows facilitate a wide field of possible applications since they provide short diffusion layers in the flow structures. Examples range from general chemical reactions (Song et al. 2006) to chemically catalyzed processes transferred from macro- to microscale like fluorination (Chambers et al. 2003; Lang et al. 2012), heterogeneous or multiphase catalysis (Kobayashi et al. 2006; Tanimu et al. 2017; Rossetti 2018), photo-catalysis (Yusuf et al. 2018), syngas production (Chen et al. 2018), extraction (Kralj et al. 2007), to mixing tasks (Wong et al. 2004) or the usage of reactions in monolith reactors (Kreutzer et al. 2005).

The great interest in downscaled liquid-liquid multiphase flows has persisted in recent years (Zhao and Middelberg 2011; Chou et al. 2015; Shi et al. 2019): The process driving forces such as pressure, temperature and concentration gradients and their resulting heat and mass transfer rates can be precisely adjusted (Haase et al. 2016; Sattari-Najafabadi et al. 2018). In combination with increased reaction rates, this can result in new process windows and often leads to an increased selectivity and yield, which delivers significant process intensification. This could mean fewer hazards and higher selectivity (Sun et al. 2016) or sustainable operation modes close to the optimal working point (Magnaudet and Eames 2000; Ern et al. 2012).

Lithographic production techniques are commonly used to fabricate microfluidic devices, which cause their crosssectional shape to be rectangular. The well-defined disperse phase (gaseous or liquid) of Taylor flows (plugs) is divided 
by continuous phase compartments called slugs. A plug does not fill the microchannel's cross section entirely. The continuous phase occupies a thin wall film and some space in the channel corners, referred to as gutters (van Steijn et al. 2009). This specific combination establishes unique flow structures and allows the continuous phase to bypass the droplet (plug) and improve mixing due to re-circulation.

The flow inside and around Taylor droplets is dominated by surface tension forces for low capillary numbers $\mathrm{Ca}=$ $\frac{u_{0} \eta_{\mathrm{c}}}{\sigma}$, where $\sigma$ describes the interfacial tension, $\eta_{\mathrm{c}}$ the dynamic viscosity of the continuous phase, and $u_{0}$ denotes the superficial velocity. The superficial velocity $u_{0}=\frac{Q_{\mathrm{tot}}}{A_{\mathrm{ch}}}=\frac{Q_{\mathrm{c}}+Q_{\mathrm{d}}}{A_{\mathrm{ch}}}$ is calculated from the total volume flow $Q_{\text {tot }}$ divided by the area of the channel cross section $A_{\mathrm{ch}}$. Herein, $Q_{\mathrm{c}}$ and $Q_{\mathrm{d}}$ represent the volume flow of the continuous and the disperse phase. The viscous forces dominate the inertia forces $\operatorname{Re}_{\mathrm{c}}=\frac{\rho_{\mathrm{c}} u_{0} H}{\eta_{\mathrm{c}}} \leq$. Here, $\rho_{\mathrm{c}}$ denotes the density of the continuous phase and $H$ is the channel height. Buoyancy forces are negligible, since the Bond number $\mathrm{Bo}=\frac{\Delta \rho g H^{2}}{\sigma} \ll 1$. Herein, $\Delta \rho$ denotes the density difference between the two phases and $g$ is the gravitational acceleration.

So far, the complex hydrodynamic interactions that relate the relative velocity of the Taylor droplets to the structures of the velocity field have not yet been entirely understood. As a consequence, the advantages of non-fluctuating Taylor flow still cannot be fully accessed. The primary influence of the $\mathrm{Ca}$-number on the droplet mobility has been reported and investigated by Jakiela et al. (2011) and Jose and Cubaud (2014). Later, Helmers et al. (2019b) developed a gray-box model to describe the Taylor droplet's relative velocity semi-analytically. In their work, the primary influence of the capillary number is successfully related to the pressure gradient that drives the bypass flow through the gutters. A single gutter of a Taylor droplet can be abstracted as a subordinate channel. Secondary factors like the gutter geometry, the wall boundary conditions and the viscosity ratio between the disperse and continuous phase appoint the hydrodynamic flow resistance of the gutter flow and consequently determine whether the pressure gradient along the gutter length is able to establish a relative flow. The role of the viscosity ratio $\lambda$ between the phases of such flow conditions has been discussed recently in experimental (Kovalev et al. 2018) and simulation works (Ransohoff and Radke 1988; Shams et al. 2018; Rao and Wong 2018).

The question, which factor determines the inner flow structure and pressure drop, like the number, shape and position of vortices, is not clarified. So far, the Ca-number (Jakiela et al. 2012; Liu et al. 2017), viscosity ratio (Ma et al. 2014; Liu et al. 2017), relative droplet velocity (Leong et al. 2016) as well as the influence of surfactants (Fuerstman et al. 2007; Angeli and Gavriilidis 2008) have been discussed: An addition of surfactants to the continuous phase leads to a lower relative droplet velocity and a surfactant load on the surface. Surfactants may absorb locally to the interfacial area (Olgac and Muradoglu 2013; Luo et al. 2019) and influence the viscosity of the interfacial area and the film thickness. Additionally, the formation of micelles leads to an increase in the bulk phase viscosity, which also influences the viscosity ratio $\lambda=\frac{\eta_{d}}{\eta_{c}}$ between the phases.

A number of $\mu$ PIV experiments have been performed in the last years, to study topological aspects of the flow field inside Taylor flows. Kinoshita et al. (2007) and Oishi et al. (2011) used high-speed confocal scanning microscopy to acquire multiple measurement planes with $2 \mathrm{D}$ velocity information that established and tested their experimental setup. Ma et al. (2014) and Liu et al. (2017) carried out measurements to address the effects of capillary number, droplet geometry, viscosity ratio and interfacial tension on the flow topology. Mießner et al. (2020) published an experimental in-depth investigation of the 3D3C velocity field in and around Taylor droplets, but did not vary any flow parameter. Recent work (Li et al. 2020; Kovalchuk and Simmons 2021) highlights the flow topology of droplets in dependence of the droplet spacing, the viscosity ratio, the droplet size, and the aspect ratio of the rectangular microchannel using $\mu$ PIV. However, a parameterized investigation of the distinct behavior of the inner re-circulation in multi-plane resolution to uncover the topological changes related to the droplet relative velocity has not been in focus so far.

In order to address the relation between the flow topology and the relative velocity of Taylor droplets, we measured the 3D2C velocity distribution inside and outside of Taylor droplets in horizontal square microchannels with a conventional multi-plane $\mu$ PIV approach. The flow topology is investigated in the central symmetry plane of the Taylor droplets and in a cross section perpendicular to the main flow direction at the core of the droplet's main vortex. Since the primary influence of the capillary number is related to the bypass flow's driving pressure gradient, all measurements are performed in the capillary regime (Sauzade and Cubaud 2013) at a constant Ca $=0.005$. Two requirements had to be realized: a) a stationary comparable droplet shape for all performed measurements at moderate Re and steady Ca (Mießner et al. 2019; Helmers et al. 2019a) and b) an equal driving pressure for the gutter flow in every considered case. The latter isolates the influence of the secondary parameters like the Re-number, viscosity ratio $\lambda$ and surfactant concentrations on the flow topology (i.e., hydrodynamic resistance of the gutters) and hence on the relative velocity of the Taylor droplet. 
Table 1 Carried out measurements. All measurements are performed at $C a=0.005$ with $1 \mu \mathrm{moll}^{-1}$ AOT added to the disperse phase for tracer particle stabilization. For further details on the relevant dimensionless quantities, as well as on the mass fractions of the flows phases for refractive index matching, see App. 6.1. Data set number (VII) has been published by Mießner et al. (2020), and details are provided in App. 6.4

\begin{tabular}{llllll}
\hline No. & $\operatorname{Re} \lambda$ & $\frac{l_{\mathrm{g}}}{W}$ & Continuous phase & Disperse phase & RIM approach \\
\hline I & 0.960 & 0.658 & Water/DMSO & n-Hexane/sunflower oil & Helmers et al. (2020) \\
II & 0.960 & 0.464 & Water/DMSO & n-Hexane/sunflower oil & Helmers et al. (2020) \\
III & 0.730 & 0.984 & Water/DMSO & n-Dodecane & Mießner et al. (2008) \\
IV & 0.692 & 0.989 & Water/DMSO/SDS & n-Dodecane & Mießner et al. (2008) \\
V & 0.310 & 0.995 & Water/DMSO/SDS & n-Dodecane & Mießner et al. (2008) \\
Vi & 0.125 & 1.016 & Water/DMSO/SDS & n-Dodecane & Mießner et al. (2008) \\
(VII) & 0.136 & 0.672 & 1-Octanol & Water/glycerol & Mießner et al. (2008) \\
\hline
\end{tabular}

\section{Material and methods}

In this section, the design of experiments, the experimental setup and the measurement method are explained. In addition, the material properties of the liquids, the particles and surfactants used are given and the image acquisition and evaluation method is explained. Finally, we state an expression for the net relative volume flow through the gutter and introduce means to quantify the topology change of the flow: The change of the Taylor droplet's main vortex diameter and streamwise position as well as the definition of a shape factor to quantify the changes of the relative downstream velocities in the main vortex's cross sections.

\subsection{Experimental concept}

The Ca-number independently determines the droplet shape from the disperse phase material properties at low capillary numbers $\mathrm{Ca} \leq 0.02$ and moderate Re (Helmers et al. 2019a). We set a fixed ratio of viscous forces to surface tension forces of $\mathrm{Ca}=0.005$ to reliably reproduce comparable cap geometries throughout this study. The remaining secondary parameters for the flow are Re, $\lambda$, the surfactant concentration and the droplet length $l_{\mathrm{d}}$. Instead of addressing the droplet length as length reference, we focused on the gutter length $1 g$, since the droplet cap geometry is flow invariant
Fig. 1 Length properties and sizes used in this work. a Lengths visible in the top view of the droplet when using a moving frame coordinate system. The inner re-circulations are shown schematically. b Rear view of the droplet along the positive $\mathrm{x}$-axis. c Side view of the droplet (only 2 measurements planes out of 14 are indicated) (a)

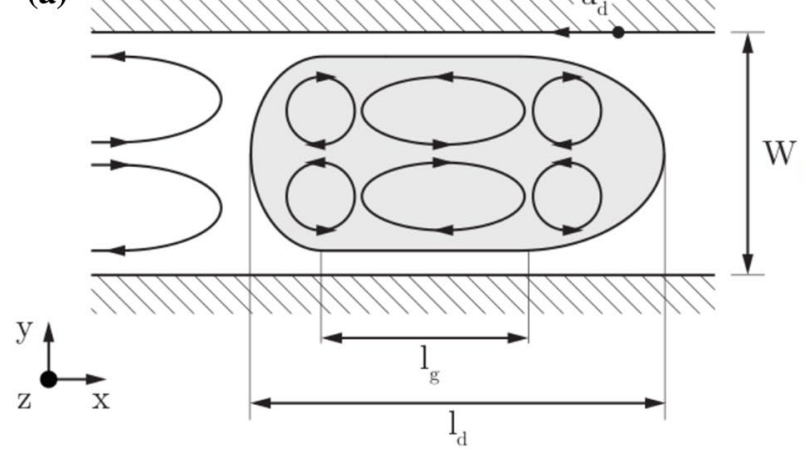

(b)

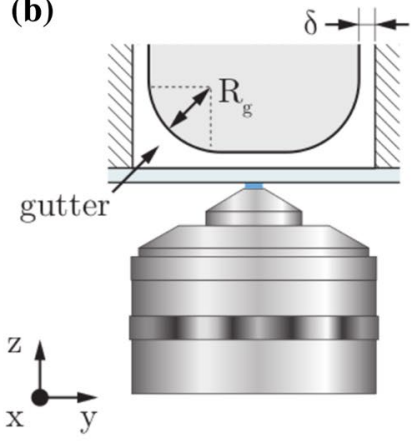

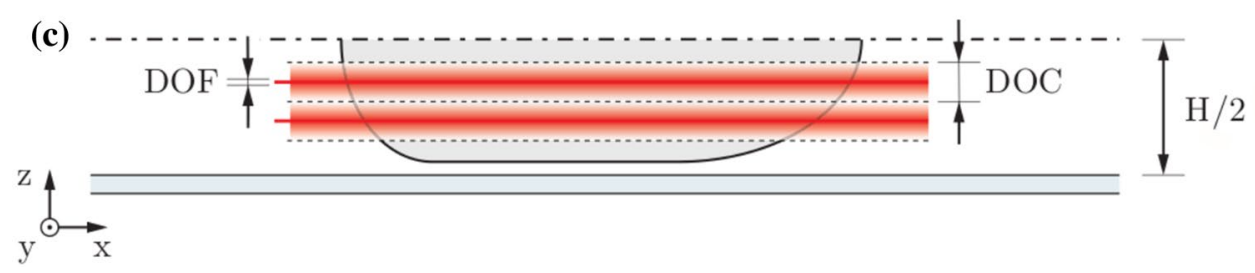


for constant $\mathrm{Ca}$ (Helmers et al. 2019a). The gutter length $\mathrm{lg}$ is the distance between the gutter entrances (Fig. 1a).

The investigation of Taylor flows by means of conventional $\mu$ PIV demands refractive index matching (RIM) of the involved phases to prevent measurement deviation due to optical distortion. However, to adjust the influential parameters, the used RIM approach has to provide a sufficient degree of freedom for parameter variation at a fixed refractive index. In combination with a variation of the mean velocity, it is possible to simultaneously perform reactive index matching, fixate the Ca-number and vary the Re-number, if two degrees of freedom are available. The viscosity ratio between the disperse phase and the continuous phase is determined by the material choice of the RIM.

Thus, either single binary mixture (Mießner et al. 2008) in combination with surfactant addition or double-binary liquid-liquid mixtures (Helmers et al. 2020) can be used to provide two degrees of freedom. For experimental reasons (particle suspension stability), we facilitated both approaches. An overview of the parameters variation is given in Table 1. Measurements I and II are performed with different droplet lengths to clarify a possible influence of the gutter length $l g$. Between measurements II to VI, the surfactant concentration is altered step-wise from 0 to $3 \mathrm{CMC}_{\mathrm{SDS}}$ as a consequence of the RIM and to evaluate the surfactant influence.

To distinguish between the influence of simultaneously varied material parameters surfactant concentration, surface tension and viscosity, we referenced the results of the surfactant-free cases II and III and used the recently published surfactant-free case VII of Mießner et al. (2020) (App. 6.4) and compare it to case VI of this study.

\subsection{Experimental Setup}

The characteristic droplets length $l_{\mathrm{d}}$, the gutter length $l_{\mathrm{g}}$, as well as the experimental setup are depicted in Fig. 1. A Taylor droplet flows with velocity $u_{\mathrm{d}}$ in a rectangular microchannel of height $H$ and width $W$. The droplet does not occupy the entire microchannel cross section, since the continuous phase remains in the channel corners (gutters). The dimension of the gutters can be described by the gutter radius $R_{\mathrm{g}}$ and the gutter length $l_{\mathrm{g}}$ (Helmers et al. 2019b).

The $x$-axis is aligned with downstream direction of the Taylor flow. The $\mu$ PIV measurement plane spans in the $x y$ direction, and its position is orthogonally varied in the $z$-direction. The velocities $u, v$ and $w$ are associated with the $x$-, $y$ - and $z$-axis, respectively. The PIV raw images contain information from the $x$ - and $y$-plane, while $z$ information is only acquired in a narrow depth. The measurements are performed at different microchannel heights $z$ to resolve the flow field in 3D. The measurement planes are distributed in the $z$-direction with a spacing $\Delta z$ that corresponds to the depth of correlation (DOC). For details on this property,

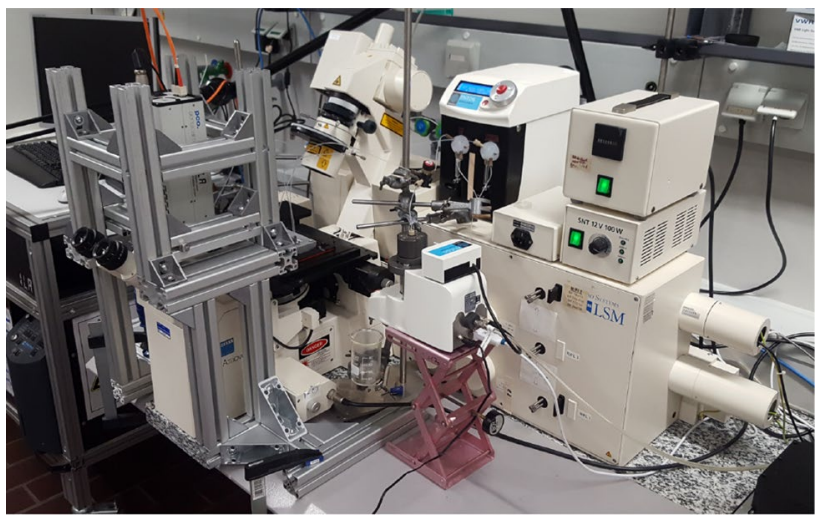

Fig. 2 Adapted inverted microscope setup Zeiss Axiovert 100 used for the conventional $\mu$ PIV measurements with peripheral equipment, PIV camera and external Nd:YAG laser

see Sect. 6.2. The experimental setup for the 3D2C- $\mu$ PIV study is similar to the design of our previous work (Helmers et al. 2020).

A Zeiss LSM410 microscope (Fig. 2) is modified such that the actual confocal scanning unit is bypassed and the attached inverted microscope (Zeiss Axiovert 100) can be used directly to perform conventional planar 2D2C $\mu$ PIV. A pulsed New Wave Research Solo-PIV III laser is guided via a laser arm into the light path of the modified system. The laser beam is precisely orientated via a motorized concave mirror to maximize the excitation light intensity, thus maximizing the fluorescence intensity yield. The laser light enters the inverted microscope and is widened by a planoconvex lens. Subsequently, a holographic diffuser equalizes the laser light to illuminate the field of view uniformly. An optical filter set, consisting of a dichroic mirror and lowpass filter, separates the excitation light wavelength from the fluorescence signal. The used microscope objective offers a working distance of $570 \mu \mathrm{m}$ at a $25 \mathrm{x}$ magnification and a depth of field DOF $=0.859 \mu \mathrm{m}$. Alternatively to the laser, a mercury vapor lamp is used to align the microfluidic devices when preparing the $\mu$ PIV measurements.

The PIV double images are recorded by a PCO.sensicam qe 670 LD 3078, which is mounted on an Carl Zeiss TV Adapter $2 / 3^{\prime \prime} \mathrm{C}$ with a $0.63 \mathrm{x}$ magnification to demagnify the image to the CCD-chip size of the camera $(6.45 \mu \mathrm{m} \mathrm{x}$ $6.45 \mu \mathrm{m}$ ). The laser timing and power, as well as the camera timing, are controlled via a timing unit. An overview of the experimental and optical parameters is given in Table 2.

Spherical polystyrene microparticles (PS-FluoRot-Fi277 from microParticles $\mathrm{GmbH}$, properties given in Table 3) are used as tracer. The particles are purchased as dry powder to exclude possible contamination caused by the preparation of a particle emulsion. The particle's fluorescence dye is adjusted to the laser wavelength $(532 \mathrm{~nm})$ to ensure a high light absorption and an intense fluorescence signal $(600 \mathrm{~nm})$. 
Table 2 Experimental and optical parameters of the experimental design

\begin{tabular}{lll}
\hline Property & Value & Unit \\
\hline Channel width $W$ & 198 & $\mu \mathrm{m}$ \\
Channel height $H$ (batch \#1) & 178 & $\mu \mathrm{m}$ \\
Channel height $H$ (batch \#2) & 192 & $\mu \mathrm{m}$ \\
Excitation wavelength & 532 & $\mathrm{~nm}$ \\
Objective magnification & 25 & - \\
Objective NA & 0.8 & - \\
Objective DOF & 0.859 & $\mu \mathrm{m}$ \\
Dichroic mirror cutoff & 552 & $\mathrm{~nm}$ \\
Longpass filter cutoff & 550 & $\mathrm{~nm}$ \\
\hline
\end{tabular}

Table 3 Properties of used fluorescent tracer particles PS-FluoRot-1.5

\begin{tabular}{lll}
\hline Property & Value & Unit \\
\hline Batch & PS-FluoRot-Fi277 & - \\
Excitation peak & 530 & $\mathrm{~nm}$ \\
Emission peak & 607 & $\mathrm{~nm}$ \\
Density & 1.05 & $\mathrm{~g} / \mathrm{cm}^{3}$ \\
Median diameter & 1.61 & $\mu \mathrm{m}$ \\
Coefficient of variation $(\mathrm{CV})$ & 2.3 & $\%$ \\
\hline
\end{tabular}

To improve the particle suspension stability, $1 \mu \mathrm{moll}^{-1} \mathrm{AOT}$ (dioctyl sulfosuccinate sodium) is added to the nonpolar continuous phase in consultation with the particle manufacturer.

The velocity profile across the entire channel cross section can be retrieved, since both flow phases are seeded with fluorescence particles. The 2D flow of the central $x y$ symmetry plane is evaluated in combination with the streamwise components of the relative velocity in the cross section through the main vortex core of the Taylor droplet.

\subsection{PIV Image acquisition and image processing}

Experimental characterization of the local flow field around droplets is preferably carried out by noninvasive measurements. Besides techniques using electric or magnetic properties of the liquids to examine the local flow field, the most prominent approaches, particle tracking velocimetry (PTV) and particle image velocimetry (PIV), are well-established optical measurement techniques. In this work, conventional $2 \mathrm{D} 2 \mathrm{C} \mu \mathrm{PIV}$ is used since it allows a higher particle seeding of the flow and therefore better spatial resolution.

To overcome the $2 \mathrm{D}$ restriction of the planar measurement technique, the measurement volume is divided into several measurement planes and a 3D measurement scan of $2 \mathrm{D}$ velocity fields is achieved with a multi-plane approach. The symmetry of the Taylor flow in straight rectangular microchannels allows to conduct measurements in one half of the channel to reduce the amount of processed data. The velocity field of Taylor droplets and its surrounding is measured at different $z$-planes at a distance $\Delta z$ of at least the $D O C=7.034 \mu \mathrm{m}$ (see Sect. 6.2). We divided half of the channel height into equidistant measurement planes to avoid recording redundant flow information. The chosen z-positions of the focal planes include the center symmetry plane and a measurement plane directly at the bottom cover of the microchannel. Since the height of the first microfluidic chip batch is $H=178 \mu \mathrm{m}$, this results in 14 measurement planes.

For the second batch $(H=192 \mu \mathrm{m})$, also 14 measurement planes are chosen to obtain integrity and comparability of the results. The orientation of the measurement planes with obtained velocity data is schematically shown in Fig. 3 with respect to the Taylor droplet interface.

To obtain a quasi-stationary flow, we performed the measurements approximately $4 \mathrm{~min}$ after starting the syringe pump. Afterwards, we considered the flow to be quasi-stationary: For the moving droplets, the flow inside and around each droplet is similar for all droplets.

Asynchronous recording requires an elevated number of 1000 double frames per measurement plane to perform a subsequent image selection and averaging step. The recording frequency of the PIV double-frame images $f_{\text {rec }}$ is limited by the used camera at $5 \mathrm{~Hz}$, while the droplet frequency oscillates around $15 \mathrm{~Hz} \pm 2 \mathrm{~Hz}$. The recording approach allows to extract a sufficient number of usable droplet images by means of image selection and preprocessing.

The temporal distance between the double frames is adjusted such that a pixel displacement of $12 \mathrm{px}$ of the tracer particles in flow regions with the highest velocity is achieved between the frames. Beginning with the center plane, the focal plane is moved to the next plane after recording 1000 images. The procedure is repeated until the measurement is completed at the bottom lid position of the microchannel.

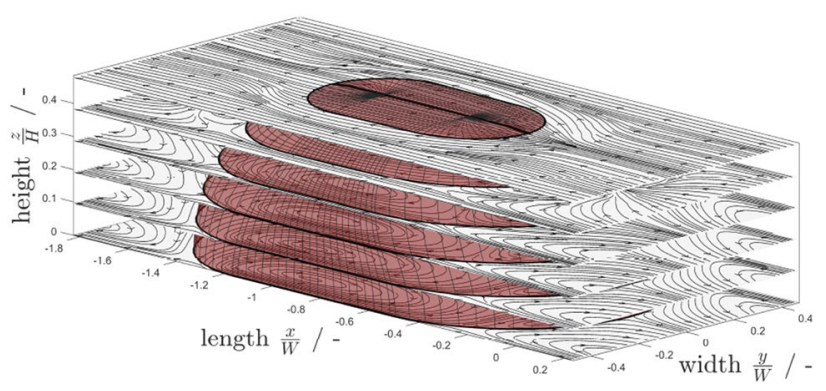

Fig. 3 Orientation of the measurement planes in $x y$-orientation with velocity data obtained from $\mu$ PIV measurements (seeding in both phases). The droplet interface is determined using the shape approximation of Mießner et al. (2019). Only 6 of the 14 measurement planes are shown to improve clarity and comprehensibility 
Due to the asynchronous recording and the length variability of the observed flow, preprocessing of the recorded data is applied to receive a quasi-stationary representation of the velocity distribution inside and around an average Taylor droplet. The preprocessing (Mießner et al. 2019) involves image selection (e.g. a statistical approach to reduce the droplet length variation) and processing (in-frame image shifting in the $x y$-direction). Since in the refractive index matched case the droplets interface cannot be discriminated optically anymore (Sect. 2.5, Fig. 5), a particle seeding gradient of the individual phases can be used to perform a threshold detection for droplet recognition and sorting (Mießner et al. 2019). Additionally, the summed images of the shifted $\mu$ PIV data can be used to determine the distance between droplet and wall for each measurement plane to retrieve the exact gutter position and distinguish between the droplet and the continuous phase in the channel corners (Fig. 4).

\subsection{Ensemble correlation PIV}

Image pairs with a defined time difference are evaluated on each measurement plane using ensemble correlation (Delnoij et al. 1999). For each measurement plane, the identified double images of suitable droplets are selected such that at least 50 image pairs are further processed (Vennemann

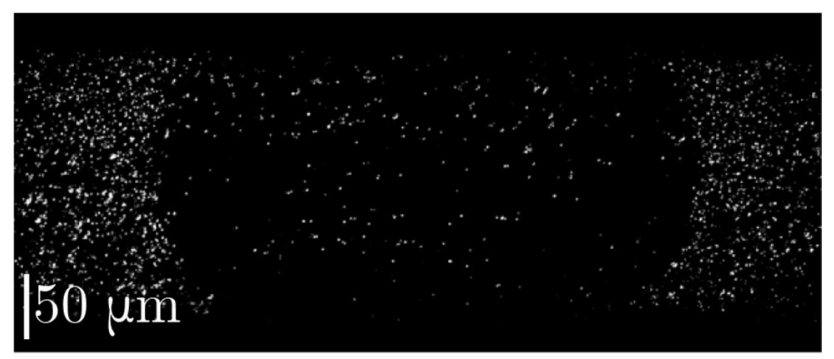

(a)

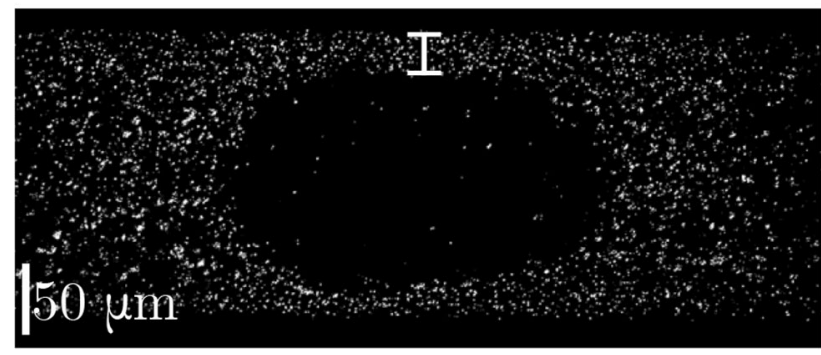

(b)

Fig. 4 Summed raw $\mu$ PIV images of sorted and shifted Taylor droplet with seeding in both phases at two measurement planes. A different seeding density in both phases allows to discriminate between droplet and continuous phase. a Sum image in the channel center plane (at channel height $z=89 \mu \mathrm{m}$ ), b sum image in a plane near the channel top wall with schematic measurement of the gutter size (at channel height $z=13.7 \mu \mathrm{m}$ )

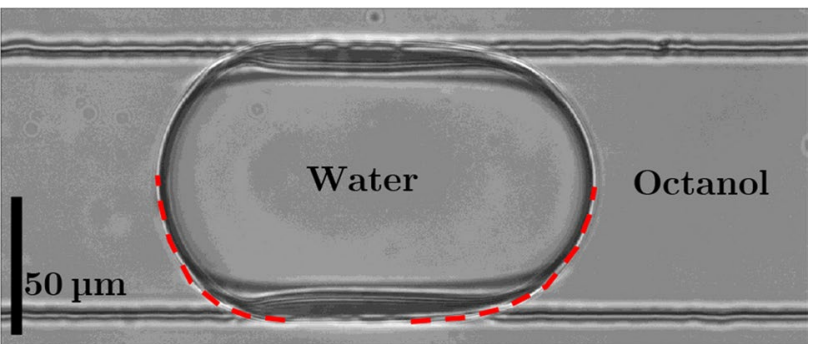

(a)

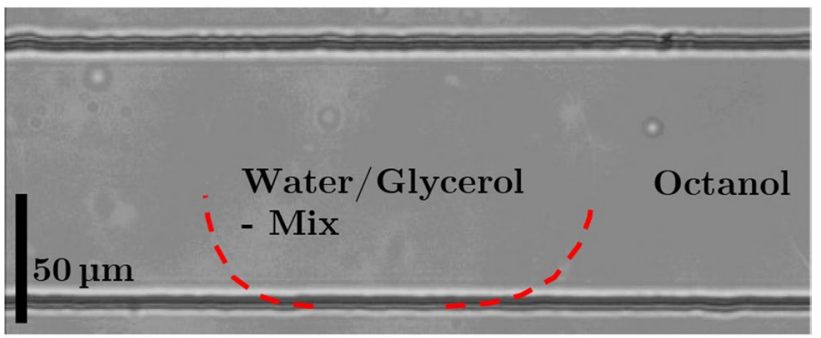

(b)

Fig. 5 Example of refractive index matching for a Taylor flow using an octanol-water/glycerol flow based on the measurements of Mießner et al. (2019). The interfacial area is marked (red dashed line). a Unmatched flow of octanol and water, b RI-matched flow of octanol and water/glycerol mixture. In this study, two RI-matching methods are applied: single-binary mixtures and double-binary mixtures

et al. 2006). To increase the data density, we made use of the flow symmetry along the center axis of the measured Taylor droplets. The selected image set is extended by additionally processing the mirrored data set in the $\mu$ PIV analysis. This results in at least 100 post-processed image pairs per measurement plane, which are subsequently evaluated with an ensemble correlation PIV algorithm. We provided a detailed description of our post-processing routine in Mießner et al. (2020).

The method relies on averaging coinciding correlation planes from a sequence of image pairs of a stationary flow (Raffel et al. 2018). After passing through the post-processing, the images fulfill the requirement of stationarity. Stochastic influences such as Brownian motion are suppressed since randomly deviated correlation peaks are averaged out in favor of the recurring correlation peaks of the stationary flow. Additionally, this technique increases the information density of the sparsely seeded flows (Adrian and Westerweel 2011).

A cycle of decreasing square interrogation window sizes (IWS) [256 128966432 16 16] px was used with an overlap of $50 \%$ applying ensemble correlation for each iteration. The resulting vector field of each cycle is used as input information for the next iteration step. The last evaluation step is performed twice to further increase the accuracy of the resulting ensemble correlated vector field. 
The depth of correlation is $z_{\text {corr }}=7 \mu \mathrm{m}$ (Olsen and Adrian 2000; Lindken et al. 2009; Rossi et al. 2010) and determined in detail in App. 6.2. Considering the influence of shear on the DOC, whereas in-plane shear increases the DOC, while to a lesser extent out-of-plane shear decreases the DOC, Olsen (2008) and Olsen (2010) introduced an approximation to describe the shear dependency of the DOC. Based on the estimation of Mießner et al. (2020) for similar flow conditions, we considered the deviation of the DOC due to shear to be acceptable and the chosen measurement plane distance to be valid.

\subsection{Refractive index matching with two degrees of freedom}

In addition to the advantage of being a noninvasive measurement technique, the nature of PIV, a planar and an optical measurement principle, requires undisturbed optical accessibility of the interrogated region. Besides the illumination intensity variation, optical distortion at the curved interfacial areas due to refraction or reflection leads to increased measurement error. To apply PIV in multiphase flow with curved interfaces, refractive index matching (RIM) between the flow phases is necessary. A broad overview of possible liquid-liquid as well as solid-liquid refractive index matching approaches is given in the work of Budwig (1994) and Wright et al. (2017).

For $\mu$ PIV measurements of Taylor droplets, approaches with one degree of freedom (Mießner et al. 2008) with a binary mixture in one phase matched to the other phase as well as with two degrees of freedom (Helmers et al. 2020) with binary mixtures in both flow phases were used. RIM approaches serving one degree of freedom are easier to describe, but solely allow matching the RI of one phase to the other. The material properties of the phases like density, viscosity and interfacial tension are fixed for the desired RI. Thus, the governing dimensionless numbers such as Re and Ca may only be parameterized hydrodynamically (superficial flow velocity) or geometrically (microchannel diameter). If there is only one degree of freedom available, the Ca number can only be adjusted by tuning the interfacial tension of the flow phases using surfactants.

In this work, for the RIM approach with one degree of freedom (single-binary mixture) n-dodecane is the disperse phase and water/DMSO is used as continuous phase to determine the surfactant influence. Albeit this approach indeed addresses $\mathrm{Ca}$ only, the use of surfactants in the continuous phase allows to identify the influence of surfactants by performing comparative measurements using the double-binary mixture approach.

\subsubsection{Single-binary mixtures and surfactants}

To determine the properties of the one-degree-of-freedom system of this work, refractive indices of several water/ DMSO mixtures are measured using a total reflection refractometer Kruess AR 2008 at $20^{\circ} \mathrm{C}$ and $589 \mathrm{~nm}$ as described in our previous work (Helmers et al. 2020). The measurements indicate that for a mass fraction of $\xi_{D M S O}=0.58$ the refractive index of the binary solution water/DMSO is matched with the RI of n-dodecane. Details are shown in the Appendix (Fig. 18b).

The effective concentration of the surfactants in the continuous phase is linked to the critical micelle concentration. Please note that at concentration beyond the CMC, surfactants alter the viscosity of the bulk liquid due to the formation of a lyotropic micelle phase. The results of the viscosity measurements are shown in App. 6.5. The increase in the bulk viscosity for concentrations below the CMC is less than $5 \%$, while for concentration beyond the CMC the bulk viscosity increases about $30 \%$. This will be considered when evaluating the measurements at higher surfactant concentrations.

\subsubsection{Double-binary mixtures}

The addition of an extra substance to the nonpolar phase leads to an RIM system with a binary mixture in both flow phases and provides an additional degree of freedom. Following our previous work, in which we proposed four double-binary mixture compositions (Helmers et al. 2020), we utilized the combination water/DMSO-n-hexane/sunflower oil to address the desired material properties (enlarged $\lambda$ with respect to the properties of the single-binary mixture approach (Sect. 2.5.1)).

\subsection{Relative volume flow through the gutter}

Helmers et al. (2019b) proposed a dimensionless representation of the relative velocity of a Taylor droplet: the excess velocity $u_{\mathrm{ex}}$. It is defined as the percentage deviation between the absolute Taylor droplet velocity $u_{\mathrm{d}}$ and the total mean velocity of the flow $u_{0}$.

$u_{\mathrm{ex}}=\frac{u_{\mathrm{d}}-u_{0}}{u_{0}}=\frac{Q_{\mathrm{g}}}{Q_{\mathrm{tot}}}+\frac{Q_{\mathrm{f}}}{Q_{\mathrm{tot}}}$.

Herein, $Q_{\mathrm{g}}$ and $Q_{\mathrm{f}}$ represent volume flow relative to the droplet motion that flows through the gutter and the wall film, respectively.

Conservation of mass implies that any net relative flow that is directed past a Taylor droplet changes the droplet velocity $u_{\mathrm{d}}$. If a net relative volume flow is directed from back to font, the droplet velocity is lower than the total mean 
flow velocity $u_{0}$ and the dimensionless excess velocity is negative. If a relative volume flow is directed from front to back, the droplet is faster than the total mean velocity of the Taylor droplet and the excess velocity is positive.

For a Taylor flow in rectangular microchannels, the volume flow through the wall film can be estimated with the relative cross-sectional area that is occupied by the film $Q_{\mathrm{f}} / Q_{\mathrm{tot}}=A_{\mathrm{f}} / A_{\mathrm{ch}}$ (Helmers et al. 2019b). The area of the film's cross section can be derived with the correlation for the wall film thickness of Han and Shikazono (2009) and the gutter radius estimated by Mießner et al. (2019). The Taylor droplet velocity $u_{\mathrm{d}}$ and the mean total velocity of the flow $u_{0}$ are measured experimentally. Thus, the dimensionless relative volume flow through the gutter can be estimated with

$\frac{Q_{\mathrm{g}}}{Q_{\mathrm{tot}}}=u_{\mathrm{ex}}-\frac{A_{\mathrm{f}}}{A_{\mathrm{ch}}}=\left(\frac{1}{\Omega_{\mathrm{g}}} \Delta p_{\mathrm{g}}\right) / Q_{\mathrm{tot}}$.

The relative gutter flow $Q_{\mathrm{g}}$ can be abstracted as a flow through a subordinate channel that is driven by a pressure difference $\Delta p_{g}$ from the droplet front to its back against the specific flow resistance of the gutter $\frac{1}{\Omega_{\mathrm{g}}}$. Helmers et al. (2019b) used the work of Ransohoff and Radke (1988) and Shams et al. (2018) to describe the resistance in the gutter and estimated the driving pressure difference with the Laplace pressure difference $\Delta p_{\mathrm{LP}}$ between the gutter entrances that is based on the geometric Taylor droplet interface approximation of Mießner et al. (2019).

$\frac{Q_{\mathrm{g}}}{Q_{\mathrm{tot}}}=\left(\left[\overline{R_{\mathrm{g}}^{2}} \frac{A_{\mathrm{g}}}{\eta_{\mathrm{c}} l_{\mathrm{g}}}\right] \sigma\left\{\frac{1}{R_{\mathrm{g}, \mathrm{b}}}-\frac{1}{R_{\mathrm{g}, \mathrm{f}}}\right\}\right) /\left(u_{0} A_{\mathrm{ch}}\right)$.

Herein, $\bar{R}_{\mathrm{g}}$ denotes a mean gutter radius (see Fig. 1b) and $\bar{R}_{\mathrm{g}, \mathrm{f}}$ and $\bar{R}_{\mathrm{g}, \mathrm{b}}$ the front and the back gutter radius, respectively. $A_{\mathrm{g}}$ is the mean cross section area of the gutter, $l_{\mathrm{g}}$ is the geometric length of the gutter, and $\beta$ is a dimensionless friction factor of the gutter that was suggested (Helmers et al. 2019b) to depend on the viscosity ratio $\lambda$ between the droplet and the bulk phase. After rearrangement, the relative volume flow through the gutter reads as follows:

$\frac{Q_{\mathrm{g}}}{Q_{\mathrm{tot}}}=\left[\frac{1}{\beta(\lambda)} \frac{1}{\left(\frac{l_{\mathrm{g}}}{W}\right)} \frac{\bar{R}_{\mathrm{g}}^{2} A_{\mathrm{g}}}{A_{\mathrm{ch}}^{2}}\right] \frac{1}{C a_{\mathrm{c}}}\left\{\frac{H}{R_{\mathrm{g}, \mathrm{b}}}-\frac{H}{R_{\mathrm{g}, \mathrm{f}}}\right\}$.

Fixating the capillary number $\left(C a_{\mathrm{c}}=0.005\right)$ determines the cap shapes of the Taylor droplets (Helmers et al. 2019a), the gutter geometry and with them the driving pressure for the gutter flow. Therefore, tuning the flow conditions of the gutter $\left(\beta(\lambda), \frac{l_{\mathrm{g}}}{W}\right)$ allows to investigate the now isolated relation between the change of flow topology and the droplet relative velocity of a Taylor flow.

\subsection{Quantification of the changes of the droplet's main vortex}

The relative position and diameter of the Taylor droplet's main vortex core change during the presented experiments. Both are accessible via streamline patterns and deliver quantifiable expressions of the change of the flow topology. In the following, we explain the quantification methods using the dimensions indicated in Fig. 6.

At first, the dimensionless relative vortex shift with respect to the geometric droplet center $l_{\text {vort,shift }}^{*}$ is described:

$l_{\text {vort,shift }}^{*}=\frac{\Delta x}{l_{\mathrm{g}}}=\frac{x(\text { core })-x\left(\frac{l_{\mathrm{d}}}{2}\right)}{l_{\mathrm{g}}}$.

Herein, $x$ (core) denotes the $\mathrm{x}$-position of the main vortex' core and $x\left(\frac{l_{\mathrm{d}}}{2}\right)$ is the $\mathrm{x}$-position of the geometric droplet center (Fig. 6). As reference length, we chose the gutter length $l_{\mathrm{g}}$ over the droplet length $l_{\mathrm{d}}$ to reference the position change, since the cap geometry is invariant to the change of droplet length at fixed flow conditions $(\mathrm{Ca})$. The changes of the vortex position may be positive as the vortex is situated downstream of the droplet center toward the droplet front as well as negative in case it shifts toward the rear of the Taylor droplet.

Secondly, the quantification of a dimensionless vortex core diameter reads as follows:

$d_{\mathrm{vort}}^{*}=\frac{d_{\mathrm{vort}}}{W}$.

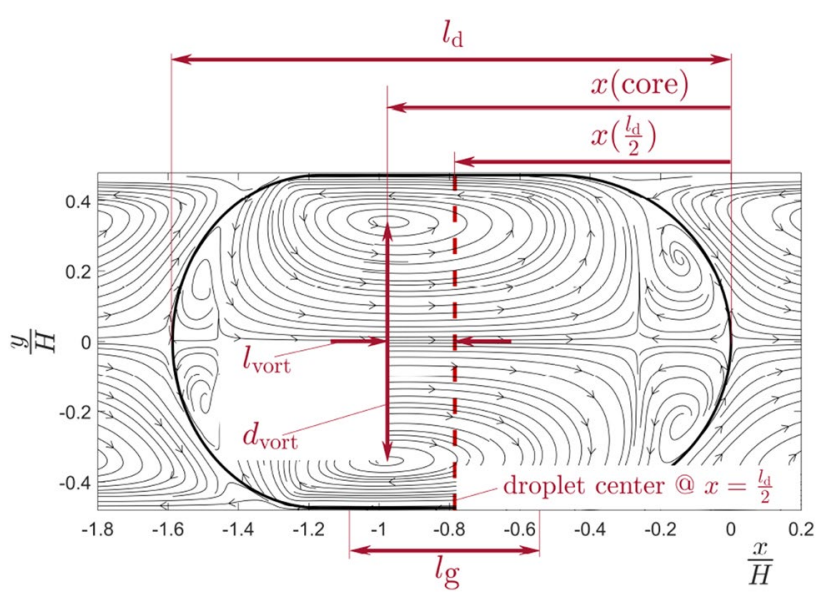

Fig.6 Quantifiable geometric indication of the change of flow topology inside Taylor droplets: The position and diameter of the center of the main droplet's toroidal vortex are described relative to the droplet's shape. Streamline pattern and interface position are taken from Mießner et al. (2020) 
Herein, $d_{\text {vort }}$ denotes the diameter at the core of the droplet's toroidal main vortex ring, while $W$ is the width of the microchannel.

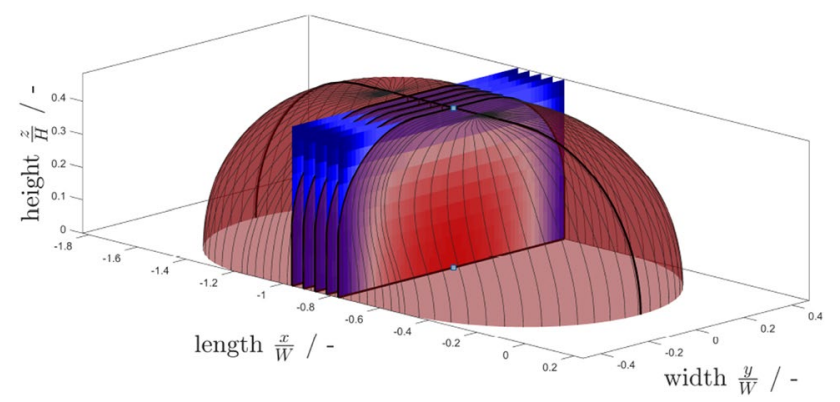

Fig. 7 Orientation of the interrogation planes in $y z$-orientation obtained from $\mu$ PIV measurements (seeding in both phases). The droplet interface is determined using the shape approximation of Mießner et al. (2019). Only 5 of the 9 planes of the averaging step around the droplet center are depicted for reasons of clarity and comprehensibility

\subsection{Definition of the topology shape factor $P$}

The considerations of the flow topology in Sect. 3.2 reveal a characteristic variation of the relative velocity distribution in the $y z$ cross-sectional plane (Jakiela et al. 2012). To quantify this effect, the relative velocity distribution in the $x$-direction

$u_{\mathrm{rel}(\bar{x}, y, z)}=u_{(\bar{x}, y, z)}-u_{\mathrm{d}}$

is determined using the stagnation point velocity of the droplet $u_{\mathrm{d}}$ at the droplet tip. The information of $9 y z$-planes around the $\mathrm{x}$-position of the droplet's main vortex are averaged (Fig. 7) to quantify the downstream motion at the core section of the main droplet vortex.

The relative velocities are normalized by the superficial velocity to retrieve the dimensionless excess velocity distribution at the cross section (yz-plane) and to ensure comparability between the measurements:

$u_{\mathrm{ex}(\bar{x}, y, z)}=\frac{u_{\mathrm{rel}(\bar{x}, y, z)}}{u_{0}}$.

The qualitative perception of the flow's topology change is based on the stagnant portion of downstream directed excess velocity distribution $u_{\mathrm{ex}(\bar{x}, y, z)} \approx 0$. The appearance of that

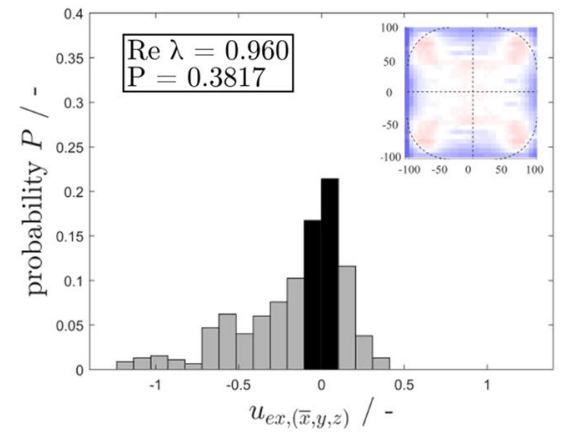

(a) Measurement I

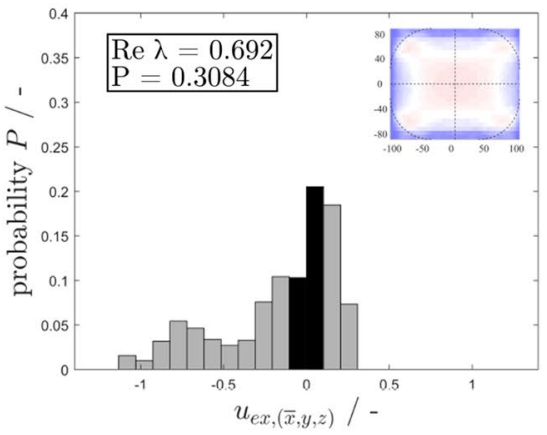

(d) Measurement IV

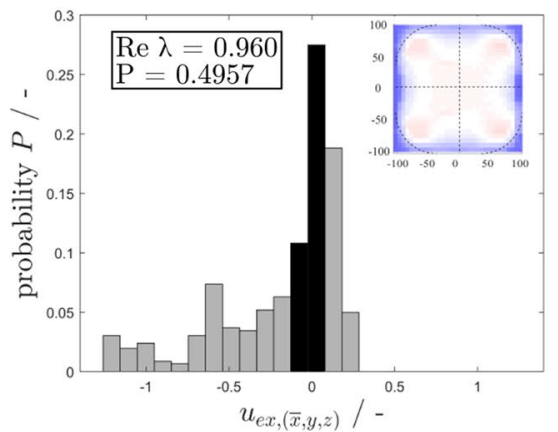

(b) Measurement II

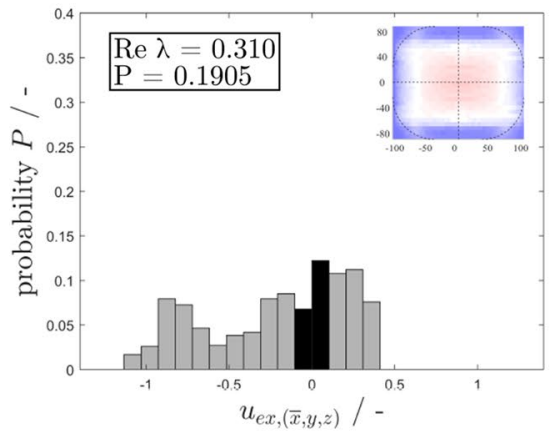

(e) Measurement V

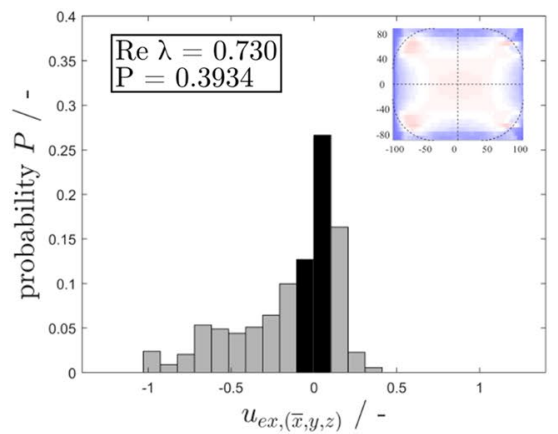

(c) Measurement III

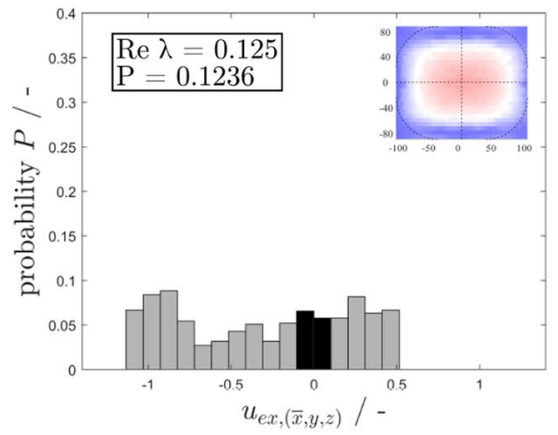

(f) Measurement VI

Fig. 8 Histograms of the probability for the excess velocity in the droplet center region. The two bins around 0 are used for the determination of the flow topology. A detailed view on the measurements is given in Sect. 3.2 
distribution changes from a cross-shaped to a circularshaped pattern. We introduced a shape factor $P$ that reliably quantifies the transition from the circular to the cross-shape flow pattern. $P$ is the fraction of the probability distribution of the excess velocity values of the flow that represents stagnation $u_{\mathrm{ex}} \approx 0$ (Fig. 8). In the investigated cross section, a circular velocity distribution pattern has a smaller circumference than a cross-shaped pattern. Thus, the probability $P$ of the stagnant fraction of the velocity distribution is low in case of the circular shape. The cross-shaped pattern has a larger perimeter, which results in an increased probability value $P$ of the stagnant fraction of the velocity distribution. For better comparison, all velocity values are analyzed in a histogram, and for all histograms, fixed binning is applied.
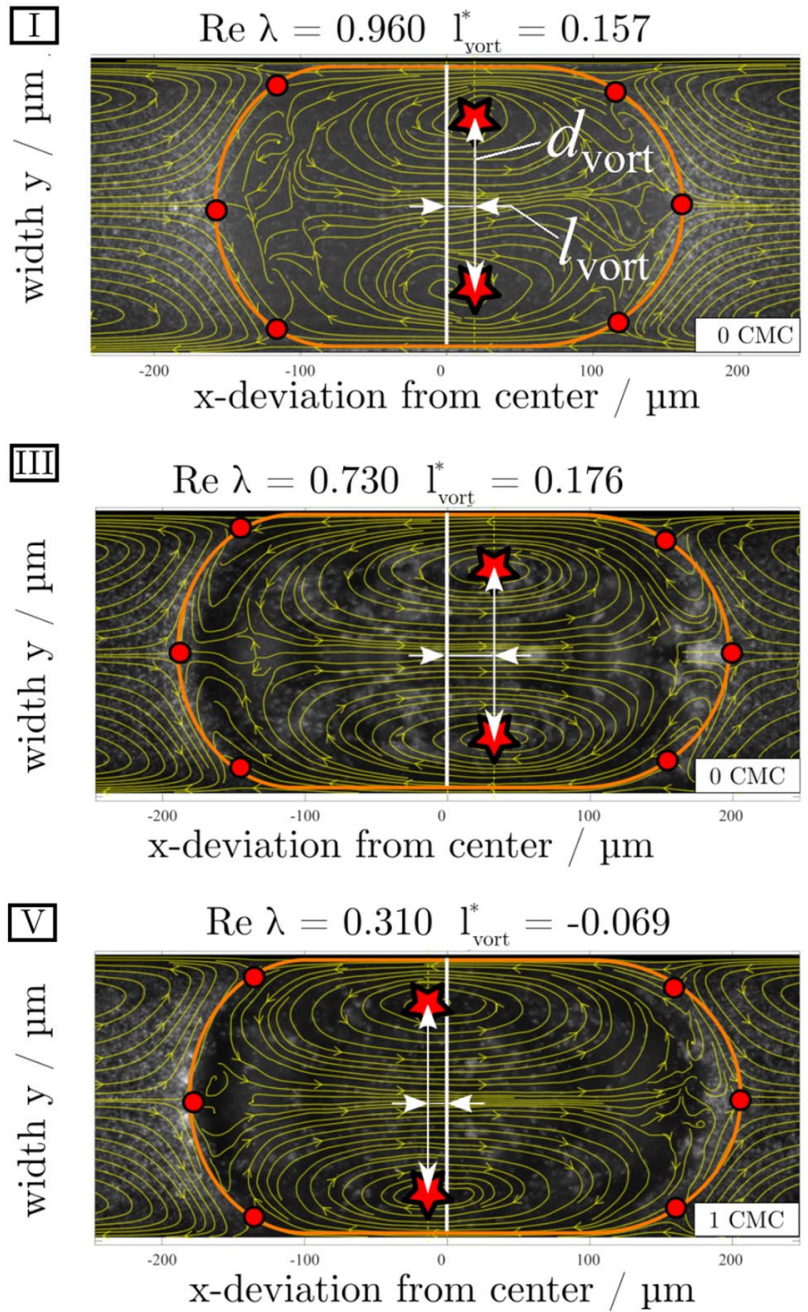

Fig. 9 Streamlines of the velocity field in the channel center plane retrieved from ensemble-averaged $\mu$ PIV measurements (velocities relative to the droplet motion in $\mathrm{x}$-direction). The corresponding particle average image in the background of the streamline plots visualizes the droplet position. The stagnation points at the interface are marked with red dots. The position of the main ring vortex as well as
Certainly, the resulting shape factor is dependent on the binning size. However, it is the intention to solely quantify and analyze the observed effect. The number of values in the two bins around 0 is used: The histograms change strongly with the presented flow cases. Thus, we considered the shape factor $P$ to be a valid measure for the purpose of quantifying the change of flow topology.

\subsection{Calculation of the shear rates}

Considering the velocity and shear information from $\mu$ PIV measurements by Mießner et al. (2020), the maximum streamwise velocity is $u_{\max } \approx 1.8 u_{0}$, while $u_{y, z} \approx 0.2 u_{0}$. The smallest transverse velocities can be found where the
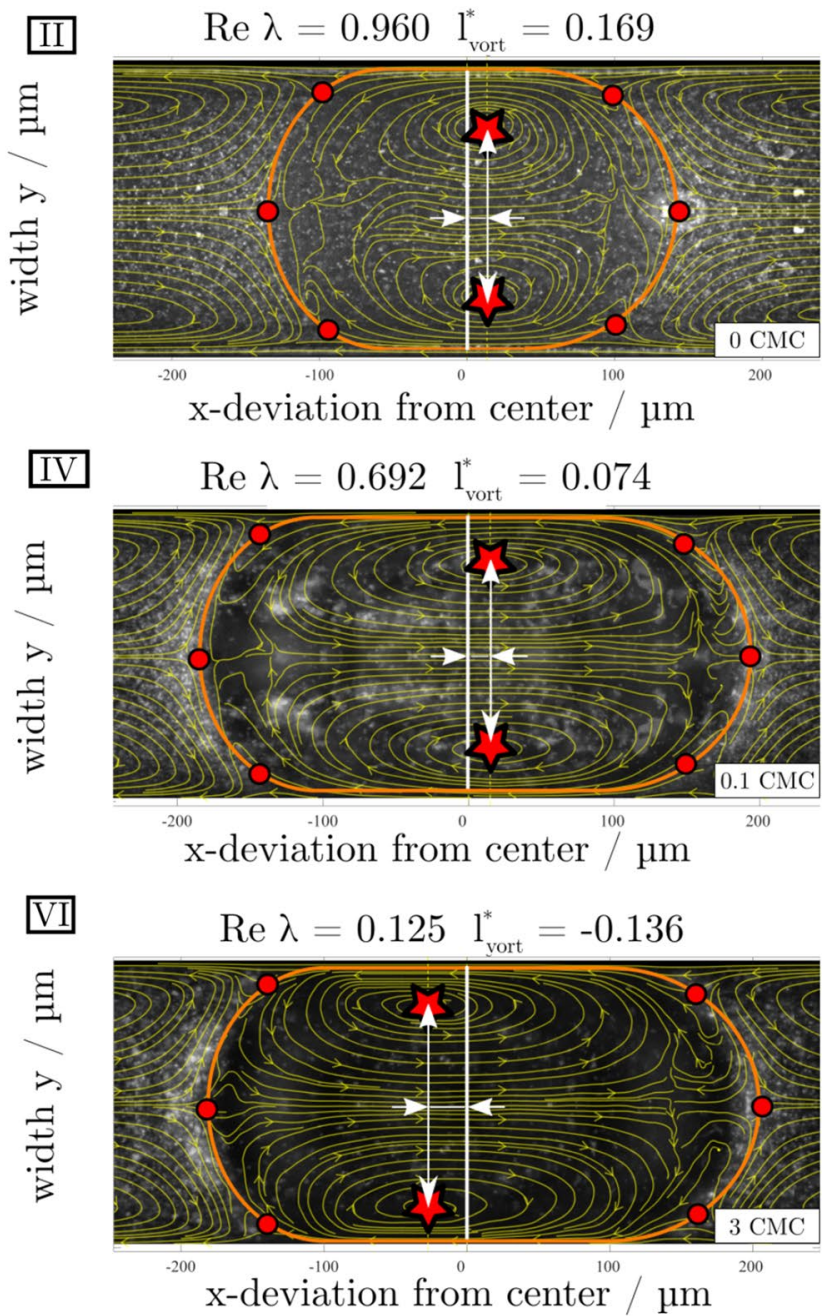

the geometrical droplet center is marked by a white line. The droplet lengths $l_{\mathrm{d}}$ are retrieved from these images. For details on the measurement parameters, see Table 1 . The shape of the droplet interface at the symmetry plane was obtained using the shape approximation of Mießner et al. (2019) and the cap curvature correlation from Helmers et al. (2019a) 
majority of the flow is directed in downstream direction. This applies for the droplet center, as the droplet is elongated and $l_{d} H^{-1}>1$. Here, it is legitimate to neglect the presence of the transversal velocity components $v, w$. We verified this assumption for the flow conditions at the main vortex center, when discussing Fig. 9 in Sect. 9

$v \ll u \rightarrow v \approx 0$

$w \ll u \rightarrow w \approx 0$.

Thus, the shear rates are evaluated using solely the axial velocity component at axial center of the main vortex core inside the Taylor droplet. The simplification allows to estimate the normalized shear distribution $\dot{\gamma}^{*}$ using 3D2C measurements without a reconstruction of the z-component.

$\dot{\gamma}^{*}=\frac{\dot{\gamma}}{\dot{\gamma}_{0}}=\frac{\left(\left|\frac{\partial \mathbf{u}}{\partial y}\right|+\left|\frac{\partial \mathbf{u}}{\partial z}\right|\right)}{\left(\left|\frac{u_{0}}{\frac{W}{2}}\right|+\left|\frac{u_{0}}{\frac{H}{2}}\right|\right)}$.

\subsection{Dimensionless indicator for momentum transfer}

The capillary number of the bulk $\mathrm{Ca}$ is the primary dimensionless quantity that governs the Taylor flow. It is reported to compare the dissipating viscous forces of the continuous phase with the driving forces of the bypass flow (Helmers et al. 2019b). A higher Ca number means a reduced flow through the bypass channel, either due to a lack of pressure gradient or due to an excess of viscous dissipation. It is this primary effect that mainly determines the change of the excess velocity of Taylor flows.

The bulk phase drives the disperse phase by means of shear via the interface of the droplet. The ratio between the droplet capillary number $\mathrm{Ca}_{\mathrm{d}}=\frac{\eta_{\mathrm{d}} u_{0}}{\sigma}$ and the bulk capillary number $\mathrm{Ca}$ is an estimate for the momentum transfer into the droplet $\mathrm{Ca}_{\mathrm{d}} / \mathrm{Ca}$. As a result, the viscosity ratio between the phases $\lambda=\eta_{\mathrm{d}} / \eta_{\mathrm{c}}$ is received as a secondary subordinate parameter.

The primary influence of the $C a$ number needs to be eliminated $(\mathrm{Ca}=$ const. $=0.005)$, in order to reveal the potential secondary influence of the Re number and $\lambda$ on the observed flow topology change and the excess velocity $u_{\mathrm{ex}}$.

We combined $R e$ and the viscosity ratio $\lambda$ to establish a secondary dimensionless indicator for the momentum transfer into the disperse phase. Re is defined by the continuous phase material parameters and the total superficial velocity of the flow. It quantifies the characteristic momentum forces in comparison with the viscous dissipation in the continuous phase that surrounds the droplets. The product $\mathrm{Re} \cdot \lambda$ indicates how much of the momentum may be coupled into the main vortex of the disperse phase: The more viscous the disperse phase is, the harder it is for the outer flow to drive the inner re-circulation.

The combination of $\operatorname{Re} \lambda$ can be rewritten to form the parameter $\mathrm{Ca}_{\mathrm{d}} \mathrm{La}$ or $\frac{\mathrm{Ca}_{\mathrm{d}}}{\mathrm{Oh}^{2}}$. Herein, La denotes the Laplace number and $\mathrm{Oh}$ states the Ohnesorge number of the continuous phase. Since $\mathrm{La}=1 / \mathrm{Oh}^{2}$, both shows the attenuation of the momentum forces and the surface tension forces by the viscous forces in the continuous phase. The capillary number of the droplet $\mathrm{Ca}_{\mathrm{d}}$ indicates the influence of the viscous forces inside the Taylor droplet. In any case, two extreme conditions emerge from this consideration:

(i) A low disperse phase viscosity (gas/liquid flows; $\lambda \rightarrow 0$ ) where the inner re-circulation is easily established by the continuous phase flow. The velocity gradients are situated in the disperse phase.

(ii) An almost infinite viscosity of the droplet $(\lambda \rightarrow \infty)$ where the droplet is almost solid and is pushed on a lubricating film with a uniform droplet velocity of $u_{\mathrm{d}}$ through the channel. In that case, little re-circulation would be induced by the continuous phase inside the droplet. Strong velocity gradients are situated in the continuous phase.

For the cases between these extremes, the droplet's main vortex is mainly induced by the relative motion of the wall. In a relative frame of reference, the relative motion of the thin wall film does not provide much space to accommodate velocity gradients and almost immediately induces the inner re-circulation of the main toroidal vortex. As a result, the velocity gradient is situated inside the disperse phase.

The spatial conditions differ strongly in case of momentum transport across the droplet interface at the gutter. At this location, there is a significant amount of continuous phase between the wall and the droplet's gutter interface. In the relative frame of reference, the momentum transfer from the moving wall to the droplet is indirectly linked via the gutter flow: The relative motion of the wall needs to transfer momentum through the continuous phase in the gutter first, before it couples into the disperse phase across the interface.

\section{Results and discussion}

To describe the change of flow topology, we visualize and quantify a shift of the main vortex core position with respect to the geometric droplet center and address a superimposed change of the main vortex core diameter. Secondly, we describe the changes of the downstream velocity distribution at the cross section through the core of the droplet's main ring vortex. Next, we analyze the cross-sectional field of shear and interpret the results in comparison with the 
velocity field. Finally, we discuss the relation between the change of the flow topology and the net flow through the gutter.

Please note that the quantification of the measured parameters suits a twofold purpose: to deliver means to observe and describe the topology change of the Taylor flow and to experimentally prove a significant correlation with the chosen dimensionless hydrodynamic parameter combination $\operatorname{Re} \lambda$. Extrapolation based on the presented correlation of the main vortex position, the main vortex diameter and the shape factor $\mathrm{P}$ in the cross-sectional flow distribution is neither intended nor valid outside the range of addressed dimensionless numbers $(C a=0.005,0.52 \leq \operatorname{Re} \leq 2.14$, $0.24 \leq \lambda \leq 2.67)$.

\subsection{Shift of central vortex position and diameter}

The position of the central vortex of Taylor droplet supplies information on the viscous energy transport and the momentum transfer between droplet, gutters, film, and the channel walls. For the investigation, we transformed the coordinate system to a moving frame description by subtraction of the stagnation point velocity at the droplet front $u_{\mathrm{d}}$ to clarify the vortex structure. We then analyzed the spatial structure of the streamline pattern of the relative velocities in the measurement plane at the microchannel center. The flow field is represented with streamlines to observe the influences of $\lambda$ and $\mathrm{Re}$ on the flow field.

The streamlines of the velocity fields are shown in Fig. 9. In the background of the plots, averaged particle raw images of the center plane allow for visual discrimination between the droplet and the continuous phase.

At the droplet's front and rear, three stagnation points are visible: At the front cap, there are two converging and one diverging stagnation point, while at the back there are one converging and two diverging stagnation points. The denomination of the stagnation points is defined with respect to the bulk phase transportation toward the interface. In case IV-VI (Fig. 9), an elevated surfactant concentration has been added to the bulk phase. A comparison of the streamlines patterns shows no obvious differences in the stagnation point position. Thus, the presence of surfactants does not interfere with the interface motion significantly.

All measurements show re-circulation of the slug region in the observed image frame at the droplet caps. Inside the Taylor droplets, a central, primary ring vortex is visible, while the expected two secondary vortices in the droplet front and rear cap (Makeev et al. 2019) are not clearly resolved. This is caused by a diminished particle seeding of the disperse phase. The insufficient seeding of the disperse phase results from increased particle deposition at inlet walls of the microchannel.
Based on the streamline distributions, two effects of a changing $\operatorname{Re} \cdot \lambda$ can be observed: The position of the main vortex' core shifts toward the droplet front with increasing $\mathrm{Re} \cdot \lambda \uparrow(\mathrm{I}-\mathrm{VI})$, and the vortex' core decreases its diameter.

Low $\mathrm{Re} \cdot \lambda \downarrow$ causes the main vortex to position closer toward the droplet's rear cap even beyond the geometric droplet centerline (V-VI). We associated the displacement of the vortex position with the variation of the viscous coupling between both phases: The stronger the droplet vortex is driven by the outer phase $(\lambda \downarrow)$, the more it is shifted toward the droplet back. Thus, if the disperse phase viscosity is low, the continuous phase dominates the flow and the main velocity gradients are situated inside the droplets (VI).

In this context, a change of vortex displacement with $\mathrm{Re} \cdot \lambda$ is visible (Fig. 10a). We concluded that the shift of the central vortex is strongly connected to the momentum transfer. A linear correlation of the position shift and $\mathrm{Re} \cdot \lambda$ follows Eq. 12 as well as supplies the coefficients $l_{1}=-0.1493 \pm 0.0263$ and $l_{0}=0.7396 \pm 0.019$ at $r=0.96$. Statistic significance is proven via an $\mathrm{F}$ test on the Pearson correlation coefficient ( $p=0.003$ ).

$l_{\mathrm{vort}, \text { shift }}^{*}=l_{1} R e \cdot \lambda+l_{0}$.

We observed that higher $\operatorname{Re} \cdot \lambda$ leads to narrower central vortices (Fig. 10b). The more the main vortex is transported to the droplet rear, the less space is available to reverse the flow near the droplet rear cap region. Thus, the vortex diameter moves inward to accommodate the relative flow reversal.

The linear correlation between the dimensionless vortex diameter and the secondary flow parameter $\operatorname{Re} \lambda$ reads as follows:

$d_{\mathrm{vort}}^{*}=d_{1} \operatorname{Re} \cdot \lambda+d_{0}$

The correlation coefficients are $d_{1}=0.3747 \pm 0.0556$ and $d_{0}=-0.1739 \pm 0.0391$ at an $r=-0.94$. Statistic significance is proven via an $\mathrm{F}$ test on the regression coefficient $(p=0.005)$.

Both correlations experimentally prove a significant relation between the flow changes and the chosen secondary parameter $\operatorname{Re} \lambda$. These two effects can be interpreted in a relative frame of reference: The formation of the droplet's flow topology depends on the amount of momentum, which can be transferred from the channel walls into the droplets. For a highly viscous droplet phase $(\lambda \uparrow)$, only the thin wall film drives the main ring vortex of the droplet. The continuous phase in the gutters develops a velocity gradient and does not contribute to the driving mechanism of the main vortex. If the continuous phase becomes more viscous $(\lambda \downarrow)$, the gutters support the momentum transfer of the wall film and the vortex is shifted to the rear of the droplet. 

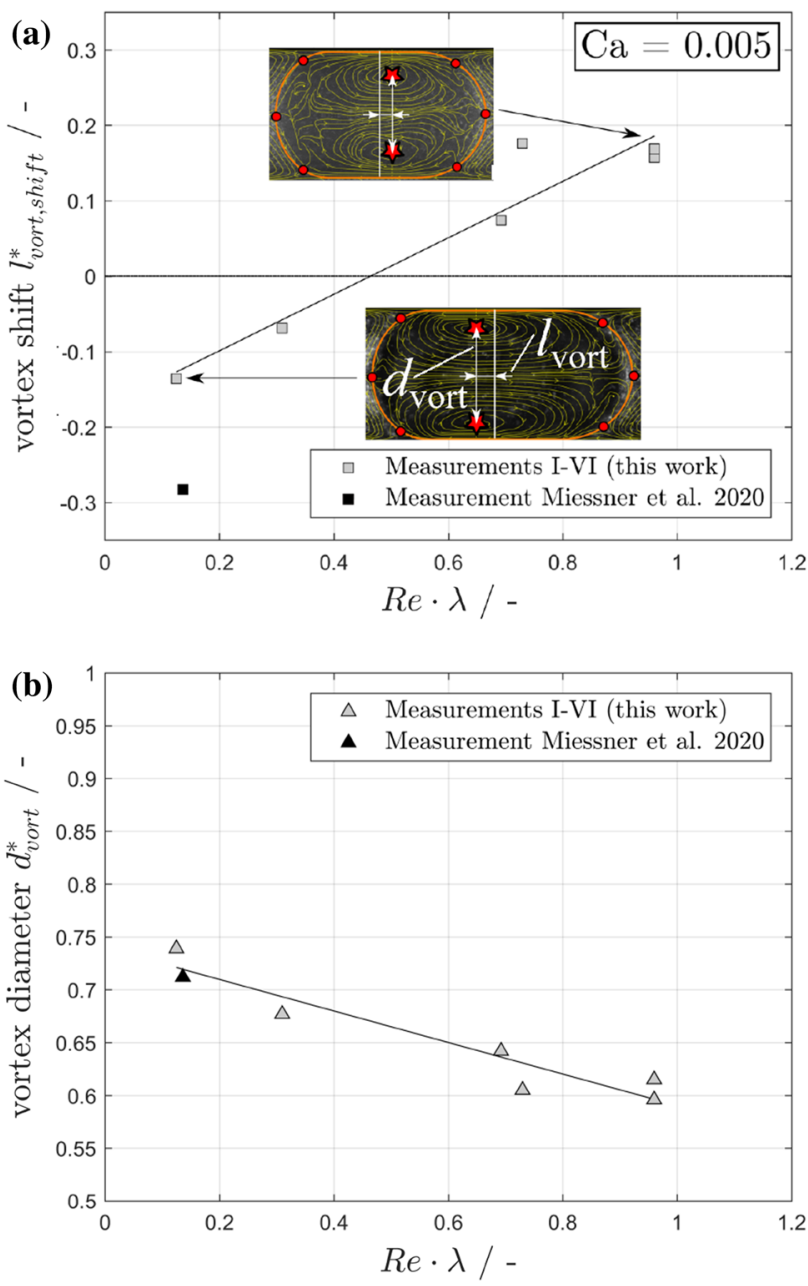

Fig. 10 Correlation between the secondary dimensionless quantity $\operatorname{Re} \cdot \lambda$ and the main vortex. Stagnation points are shown as red circles, and the vortex center as red star. The vortex shift is depicted as a white arrow. a Relative shift of the main toroidal vortex in the $x y$ symmetry plane. The shift of the main vortex is strongly connected to the momentum transfer $\mathrm{Re} \cdot \lambda$. The correlation follows $l_{\text {vort }}^{*}=l_{1} \operatorname{Re} \cdot \lambda+l_{0}$ with the coefficients $l_{1}=-0.1493 \pm 0.0263$ and $l_{0}=0.7396 \pm 0.019$. b A decrease in the main vortex relative diameter. The more the main vortex is transported to the droplet rear, the less space is available to reverse the flow near the droplet rear cap region and the vortex gives way: Its diameter moves inward to accommodate the relative flow reversal. The correlation follows $d_{\text {vort }}^{*}=d_{1} \operatorname{Re} \cdot \lambda+d_{0}$ with the coefficients $d_{1}=0.3747 \pm 0.0556$ and $d_{0}=-0.1739 \pm 0.0391$. Both correlations experimentally prove a significant connection between the changes of the main vortex and the secondary flow parameter $\mathrm{Re} \cdot \lambda$. The measurement of Mießner et al. (2020) is added to the diagram to emphasize the independence of the effect from the surfactant concentration

In case of low inertia forces ( $\operatorname{Re} \downarrow$ ), it is the viscosity of the continuous phase that dominates the droplet re-circulation. The more the inertia forces rise $(\operatorname{Re} \uparrow)$, the less important is the viscous dissipation of the continuous phase and the main vortex is mainly driven by the relative motion of the wall film.
Based on the above findings, we chose the position of the vortex core to investigate the flow-dominating downstream velocity distribution through the cross section of the microchannel at the cross section of the main vortex core. We report the change of the flow topology in Sect. 3.2.

\subsection{Flow topology in yz cross-sectional plane}

We used the velocity data retrieved by means of $\mu$ PIV to visualize flow profiles in the above-identified droplet cross sections at the core position of the Taylor droplet's main ring vortex. We determined the mean downstream velocity at the frontal stagnation points and calculated a field of relative velocities pointing in streamwise direction. The resulting cross-sectional flow patterns are shown in Fig. 11.

The work of Jakiela et al. (2012) and Rao and Wong (2018) suggests that the flow profile changes with $\lambda$. We found that the flow topology changes from a cross-shaped (I) pattern at high $\mathrm{Re} \cdot \lambda$ to a circular-shaped flow pattern at low $\mathrm{Re} \cdot \lambda(\mathbf{V I})$. A decreasing viscosity ratio and decreasing inertia influence change the momentum coupling between the phases.

At higher $\mathrm{Re} \cdot \lambda$, the relative velocity tends to point downstream (red) in close proximity of the interfacial area of the gutters. Inside the droplet, four positively oriented flow filaments at the channel corners in the gutters are visible (Fig. 11, I-IV). The continuous phase is not able to transfer the momentum from the relative motion of the gutter wall into the droplet $(\lambda \uparrow)$. The velocity gradient is located closer to or even inside the gutter. The resulting flow pattern of downstream velocities in a cross section at the vortex core has a cross-shape.

Decreasing $\mathrm{Re} \cdot \lambda$ (measurements $\mathrm{V}-\mathrm{VI}$ ), the positive flow is intensified in the channel center, while the flow near the gutters decreases. Additionally, the regions of flow inversion with respect to the droplet velocity (shown as white flow areas) are moved toward the channel center. This can be interpreted as a decrease in the hydrodynamic cross section, which is especially visible, when the local shear rates are examined (Sect. 3.3). This effect is not caused by the surface activity of the surfactants added IV-VI: The surfactant-free measurement of Mießner et al. (2020) at a similar $R e \cdot \lambda$ shows the same flow topology (App. 6.4). Thus, we attributed the correlation to the surfactant concentration solely to the viscosity increase in the continuous phase due to the buildup of a lyotropic phase. The resulting flow pattern of downstream velocities in a cross section at the vortex core has a circular shape.

To quantify the change from $\mathrm{x}$-shaped to circularshaped flow pattern, we characterized the flow topology in the $y z$ cross section with the probability distribution of low relative velocities (white areas in Fig. 11) and retrieved a 


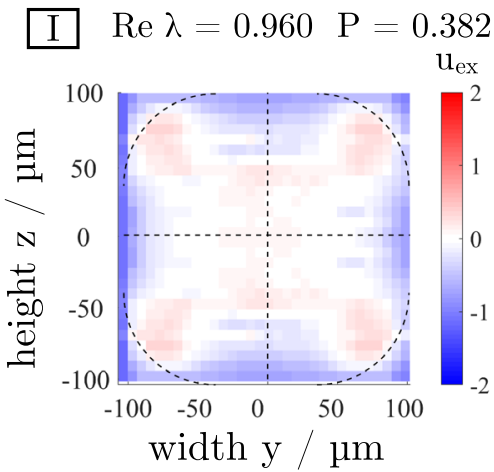

IV $\operatorname{Re} \lambda=0.692 \mathrm{P}=0.308$ $\mathrm{u}_{\mathrm{ex}}$

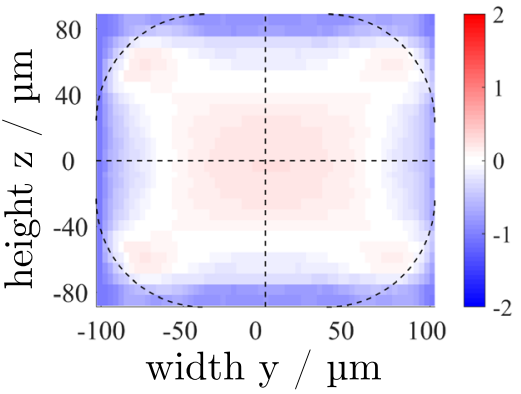

II $\operatorname{Re} \lambda=0.960 \mathrm{P}=0.496$ $\mathrm{u}_{\mathrm{ex}}$

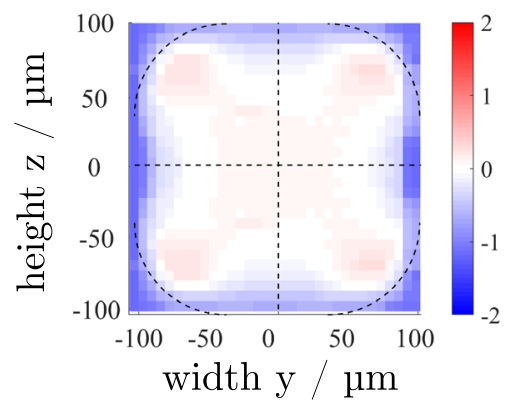

$\mathrm{V} \operatorname{Re} \lambda=0.310 \mathrm{P}=0.191$

$\mathrm{u}_{\mathrm{ex}}$

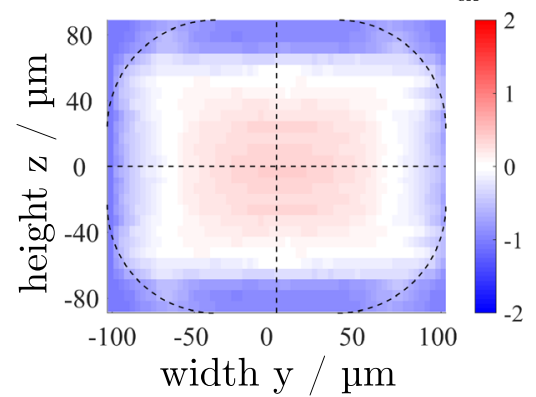

III $\operatorname{Re} \lambda=0.730 \quad \mathrm{P}=0.393$

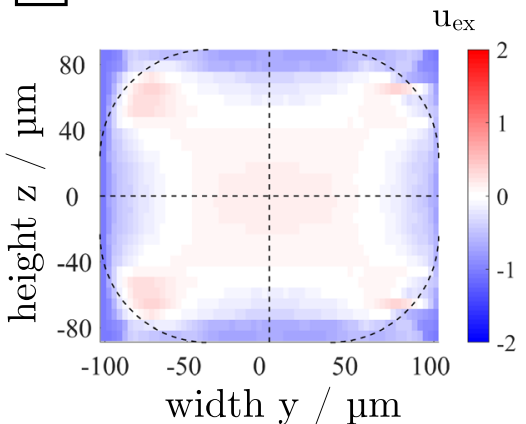

VI $\operatorname{Re} \lambda=0.125 \mathrm{P}=0.124$

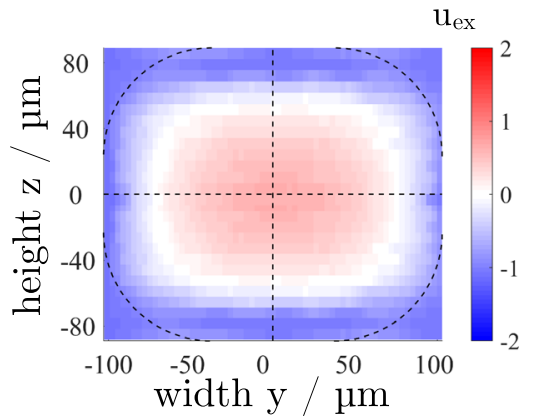

Fig. 11 Cross-sectional front view on the droplet (yz-plane) at the main vortex center. The local excess velocities $u_{\text {ex,loc }}$ for the entire cross section are depicted. Red volume flows point downstream in the positive $x$-direction (toward the droplet front and the observer); blue volume flows point in the opposite direction. For details on the measurement parameters, see Table 1 . A comparison with the data set (VII) of Mießner et al. (2020) is provided in App. 6.4

quantitative shape factor $P$ (Sect. 2.8). A rising probability of low relative velocities can be interpreted as a growing outer perimeter of the specific flow structure (x-shape). The quantified relation between the shape factor $P$ and the parameter for momentum coupling $R e \cdot \lambda$ is shown in Fig. 12. Our measurements experimentally show a linear correlation for the shape factor $P$ with the momentum coupling according to the equation.

$P=p_{1} \operatorname{Re} \cdot \lambda+p_{0}$.

The coefficients are $p_{1}=0.4352 \pm 0.0500$ and $p_{0}=0.0572 \pm 0.0319$. The Pearson correlation coefficient $r=0.98$ and a $\mathrm{F}$ test on the correlation coefficient ( $p=0.003$ ) supports the statistic significance. Since the correlation is in good agreement with the data reported by Mießner et al. (2020), who utilize a quasi-surfactantfree material system to apply Taylor flows at low $\operatorname{Re} \lambda$, we assumed this correlation to be independent from surface activity effects. The surfactant concentration in the continuous phase solely increases the bulk viscosity due to the formation of a lyotropic phase.

Phenomenologically, we interpreted the observation as follows: Since the main ring vortex of Taylor droplets is 
generally driven by the motion of the interface, we needed to differentiate between a wall-film-based momentum transfer and the transfer across the interface of the gutters:

(i) The direct and dominant momentum transfer takes place between the wall film and the droplet's main vortex. The film thickness is too small to accommodate velocity gradients and directly passes the relative motion of the wall into the Taylor droplet.

(ii) The motion of continuous phase through the gutters strongly depends on the flow conditions and on the viscosity ratio.

If the liquid in the gutter is highly viscous compared to the droplet viscosity $(\lambda \downarrow)$, the channel wall in the gutter transfers momentum easily to the gutter interface and into the droplet. In this case, the momentum transfer via the gutter interface is as efficient as the wall-film-based momentum transfer. The according velocity gradient is situated in the droplet, shifts the vortex core to the droplet rear and results in the circular shape of the observed topology.

If the liquid in the droplet is highly viscous $(\lambda \uparrow)$, then the wall-induced momentum in the gutter cannot be transferred to the droplet interface. An almost solid droplet establishes a velocity gradient in the gutter and establishes the $\mathrm{x}$-shape of the flow topology.

At low Re, the damping viscous forces in the continuous phase dominate the flow through the gutter. In an absolute frame of reference, the bulk phase in the corners can be set into motion by the moving droplet and its momentum transfer across the interface. Elevated viscous forces of the continuous phase result in a decreased absolute fluid mobility inside the gutter and can be considered at the same time as an increased relative fluid transport from the droplet front to its rear. The backward-directed fluid transport supports the backward motion of the wall film and establishes the observed circular shape of the topology.

At higher Re, inertia dominates the viscous damping of the continuous phase. The more pronounced the inertia forces are in the bulk, the easier it is to move the liquid in the corner in an absolute frame of reference, which translates to reducing the mobility of the bulk in the relative frame. Thus, the wall-film-driven mechanism is stronger than the decreased influence of the gutter and the $\mathrm{x}$-shape of the topology is created.
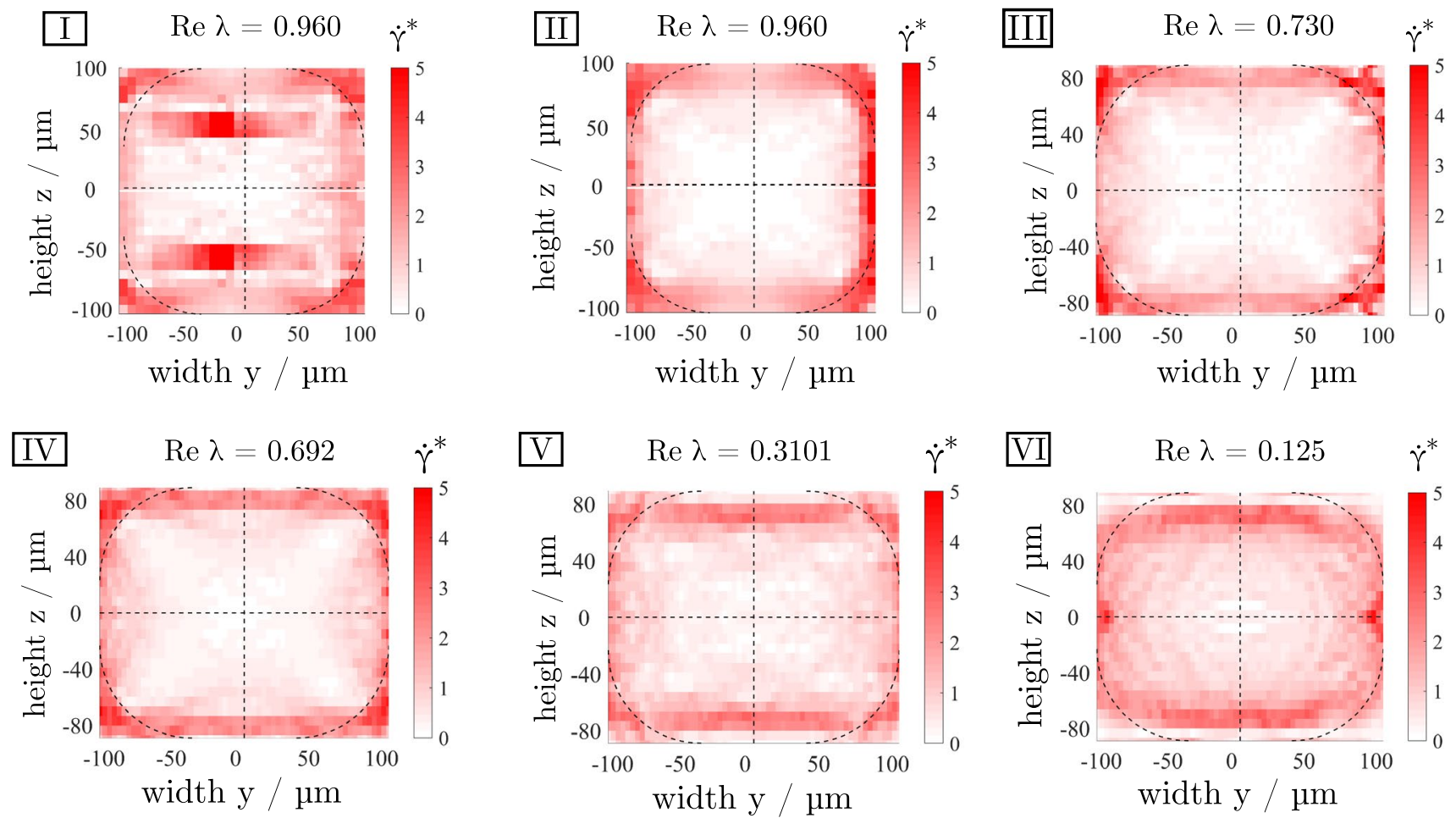

Fig. 13 Front view on the droplet in yz-plane at the main vortex center (right side). The local normalized shear rates are calculated as described in the text. For measurement $\mathbf{I}$, the artifacts lead to strong signal noise at $z \approx 0.25 H$. For $\lambda \rightarrow 0$, the main shear is dissipated on the inner side of the interfacial area. At $\lambda>0.3$, higher shear rates can be obtained in the gutters 


\subsection{Cross-sectional shear rate distribution}

Besides the velocity information, the amount of dissipated energy can be quantified by a consideration of the shear rate distribution of the flow. This allows to identify regions of high dissipation and discriminate whether the main dissipation takes place in the continuous phase or in the droplet. The results of the calculation of the shear rates (Sect. 2.9) are shown in Fig. 13. In general, the highest shear rates occur in the less viscous flow phase.

For larger and intermediate values of $\mathrm{Re} \cdot \lambda$ (measurements $\mathbf{I}-\mathbf{I V}$ ), the main shear is located in the continuous phase of the gutters. The liquid in the gutters is driven by the motion of the viscous body of the droplet. The flow utilizes the entire hydrodynamically available channel cross section. This coincides with the results of Ransohoff and Radke (1988), Shams et al. (2018) and Helmers et al. (2019b), who found that a larger viscosity ratio $\lambda$ leads to increased dissipation in the gutters.

With decreasing $\operatorname{Re} \cdot \lambda$, a transition of the areas with a higher shear rate can be identified. For low $\mathrm{Re} \cdot \lambda$ (measurements $\mathbf{V}-\mathbf{V I}$ ), the main shear is located in the droplet. The viscous liquid in the gutter sticks to the channel walls and supports the wall film in driving the main droplet vortex. The velocity gradient is located inside the droplet. In contrast to the measurements at larger $\operatorname{Re} \cdot \lambda$, the available hydrodynamic diameter is reduced by the viscous gutters. The lower $\mathrm{Re} \cdot \lambda$, the smaller is the effective hydrodynamic diameter and the more increases the excess velocity of the droplet (Helmers et al. 2019b).

\subsection{Influence of the flow topology on the gutter flow}

In Sect. 2.6, we explain the direct connection of the net volume flow that bypasses the Taylor droplet through the gutter $\frac{Q_{g}}{Q_{\text {tot }}}$ and the dimensionless expression of the excess velocity of the droplet $u_{\mathrm{ex}}$. We use this relation in this section to discuss the influence of the topology change with the momentum coupling $\mathrm{Re} \cdot \lambda$ on the net relative volume flow through the gutter.

At first glace, we found no obvious relation between the momentum coupling $\mathrm{Re} \cdot \lambda$-related flow topology change and the net volume flow that bypasses the Taylor droplets through the gutter (Fig. 14a). Small values of Re $\cdot \lambda$ as well as elevated values of $\mathrm{Re} \cdot \lambda$ show large net gutter flows. Considering only the Taylor droplet of the same gutter length $\frac{l_{g}}{W} \approx 0.996$ (gray area) suggests a trend of decreasing gutter flow with increasing momentum coupling $\mathrm{Re} \cdot \lambda$. However, we cannot substantiate the significance of a linear trend with an $\mathrm{F}$ test. Based on the gutter length, it is possible visually
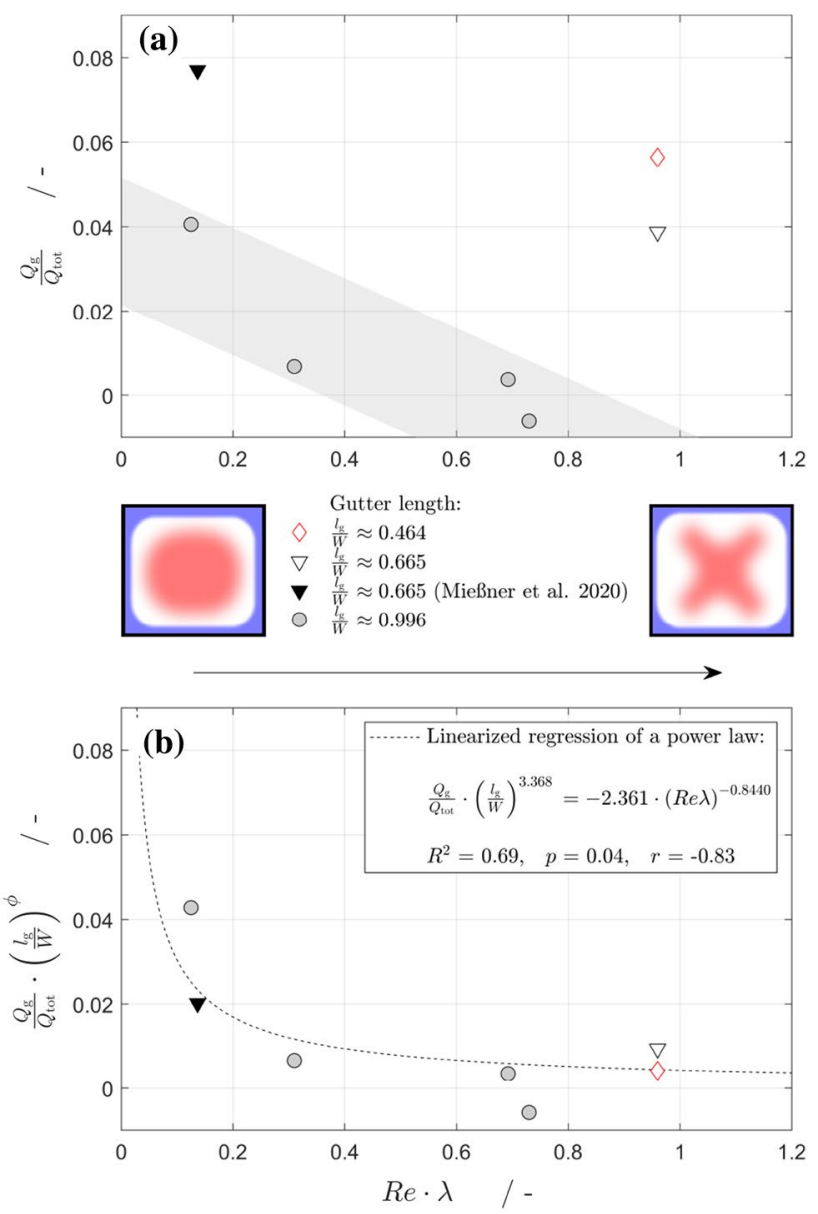

Fig. 14 Relation between the net volume flow that bypasses a Taylor droplet through the gutter $\frac{q_{\mathrm{g}}}{Q_{\text {tot }}}$ and the change of the flow topology due to the momentum coupling $\mathrm{Re} \cdot \lambda$ (circular shape toward $\mathrm{x}$-shape). a A direct relation of the quantities is not obvious. Following the visual trend of the droplets with a gutter length of $\frac{l_{g}}{W} \approx 0.996$ (gray area), the gutter flow reduces with increasing momentum coupling $\operatorname{Re} \cdot \lambda$. However, the postulated linear trend has no significance when being F-tested. b Scaling the gutter flow data with the gutter length $\frac{g_{g}}{W}$ exhibits a power law-based relation. A linearization of the power law allows the application of the $\mathrm{F}$ test which supports the significance of the trend

to group the data. However, the data basis is insufficient to be conclusive in this representation.

We used Eq. 4 to justify the scaling of the gutter flow $\frac{Q_{\mathrm{g}}}{Q_{\mathrm{tot}}}$ with the gutter length $\frac{l_{g}}{W}$ and optimize it for a linear representation of the entire data set (Fig. 14b). As a result the gutter length receives the exponent $\phi=3.368$. However, the postulated linear overall trend carries also no $F$-test-based significance.

Judging from the scaled data set and based on literature approaches (Ransohoff and Radke 1988; Shams et al. 2018; Helmers et al. 2019b), we assumed that the scaled data set is a nonlinear function of $\operatorname{Re} \cdot \lambda$ and follows a power law 
(Fig. 14b), dashed line). The linearized regression of the power law allows to conduct an F test that supports the significance of the estimated relation. The regression coefficient $R^{2}=0.69$, the $\mathrm{p}$ value is below the chosen threshold of 0.05 , and the Pearson regression coefficient is $r=-0.83$. Thus, the relation between the net gutter flow and the flow topology is likely to follow a power law that depends on the parameters $\frac{l_{\mathrm{g}}}{W}, \operatorname{Re}$ and $\lambda$.

Equation 4 shows a linear relation of the gutter flow and the gutter length. The viscosity ratio between the droplet and the bulk phase is incorporated into the dimensionless friction factor of the gutter $\beta$ (see Sect. 2.6). Based on the work of Ransohoff and Radke (1988), Shams et al. (2018) and Helmers et al. (2019b), we expected the additional dependence on the gutter length $\frac{l_{\mathrm{g}}}{W}$ and $R e$ to be attributed to the dimensionless friction factor $\beta$. We suggested the following functional relation for the dimensionless friction factor $\beta$.

$\beta=c_{1}\left(\frac{l_{\mathrm{g}}}{W}\right)^{c_{2}}(\operatorname{Re} \cdot \lambda)^{c_{3}}$.

An increased momentum coupling $\mathrm{Re} \cdot \lambda$ between the bulk and the droplet phase leads to a shift of the velocity gradient into the gutter. As a consequence, the net relative volume flow through the gutter from the droplet front to its back is suppressed (Fig. 11). This means that, in an absolute frame of reference, a fast viscous droplet moves the fluid in the gutter in droplet flow direction and overcomes the stagnation of continuous phase in the gutter. The latter of which basically constitutes the net relative volume flow of the bypass through the gutter.

A low momentum coupling $\mathrm{Re} \cdot \lambda$ evokes a velocity gradient that is clearly situated inside the Taylor droplet, which leads to a stagnation of continuous phase in the gutter in the absolute frame of reference. Translated into the relative droplet coordinates, this leads to an increase of the net relative flow through the gutter toward the back of a Taylor droplet. As a consequence, the dimensionless excess velocity of the droplet $u_{\text {ex }}$ increases.

The elevated influence of the gutter length $\frac{l_{\mathrm{g}}}{W}$ could be related to the development of the flow profile along the gutter. With increased momentum coupling $\operatorname{Re} \cdot \lambda$, the overall shape of the gutter gains importance since the cross section of the gutter decreases toward the rear gutter exit. The cross-sectional change of the gutter is only important at high momentum coupling: In addition to the velocity gradient shift into the gutter, the tapering gutter cross section prevents the development of even steady velocity profile inside the gutter. The effect is stronger with high momentum coupling and loses importance with low energy flow.

Please note that the data basis of this work is not sufficient to validate a correlation. However, we suggest an adapted description for the dimensionless net relative volume flow through the gutter $\frac{Q_{\mathrm{g}}}{Q_{\text {tot }}}$ that is based on the results of this work. In order to reduce multiple dependencies on the absolute mean flow in $\mathrm{Re}$ and $\mathrm{Ca}$, we expanded Eq. 4 with $\frac{\mathrm{Ca}^{c_{3}}}{\mathrm{Ca}^{c_{3}}}$ and exchange $\frac{R e}{C a}$ with $\frac{1}{O h^{2}}$ that resents a sole material dependency. As a consequence, the influence of the $\mathrm{Ca}$ changes accordingly to $C a^{\left(1+c_{3}\right)}$.

$$
\begin{aligned}
\frac{Q_{\mathrm{g}}}{Q_{\mathrm{tot}}}= & {\left[\frac{\mathrm{Oh}^{\left(2+c_{3}\right)}}{c_{1}\left(\frac{l_{\mathrm{g}}}{W}\right)^{(\phi-1)} \lambda^{c_{3}}} \frac{1}{\left(\frac{l_{\mathrm{g}}}{W}\right)} \frac{\bar{R}_{\mathrm{g}}^{2} A_{\mathrm{g}}}{A_{\mathrm{ch}}^{2}}\right] \cdots } \\
& \ldots \frac{1}{\mathrm{Ca}^{\left(1+c_{3}\right)}}\left\{\frac{H}{R_{\mathrm{g}, \mathrm{b}}}-\frac{H}{R_{\mathrm{g}, \mathrm{f}}}\right\} .
\end{aligned}
$$

Aiming at constant process conditions in a continuous droplet chain, one needs to avoid a distribution of droplet velocity. The net transport of bulk phase relative to the Taylor droplets needs to be suppressed. The transport through the wall film cannot be influenced easily, since it is determined by the film thickness. However, the net flow through the gutter can be reduced by tuning the following parameters of the dimensionless friction factor $\beta$ : increased gutter length $\frac{l_{\mathrm{g}}}{W}$ (long Taylor droplets), increased viscosity ratio $\lambda$ between droplet and bulk and a small Ohnesorge number $O h$ of the continuous phase.

\section{Conclusion}

This work investigates the relation between the relative velocity of Taylor droplets and the change of the flow topology of their velocity fields. For this purpose, 3D2C velocity fields of Taylor droplets moving in rectangular microchannels have been measured by means of multiplanar $\mu$ PIV at a narrow depth of correlation of $7 \mu \mathrm{m}$ and a constant $C a=0.005$. An ensemble-averaging approach in combination with an improved droplet sorting routine was used to carefully retrieve velocity information at different height positions. A one-degree-of-freedom as well as a novel twodegree-of-freedom approach was used to perform refractive index matching.

Based on these measurements, the vortex position and flow profile in the droplet cross section were examined depending on the parameter $R e$ and $\lambda$. The flow topology proposed in the recent literature has been experimentally validated, and the influential parameters were identified as the viscosity ratio $\lambda$ and $R e$. Neither an immobilization of the interface due to the surfactants could be observed, nor a position change of the stagnation points. The flow field appeared to be invariant to the surface activity. A 
straightforward influence of surfactant concentration beyond a lyotropic viscosity increase could not be shown.

A change of the position and diameter of the main vortex core as well as the flow topology change was experimentally identified depending on the combination of $\operatorname{Re}$ and $\lambda$. The combined parameter can be used to form $\operatorname{Re} \lambda=\frac{\mathrm{Ca}_{\mathrm{d}}}{\mathrm{Oh}^{2}}$. The capillary number of the droplet $\mathrm{Ca}_{\mathrm{d}}$ indicates the influence of the viscous forces inside the Taylor droplet, and $\mathrm{Oh}$ describes the attenuation of the momentum forces and the surface tension forces by the viscous forces in the continuous phase. The interplay of these factors determines the momentum transfer.

Increased momentum coupling into the Taylor droplet $\mathrm{Re} \cdot \lambda$ causes the cross-sectional distribution of downstream velocities to change their topology: from droplet center axis concentration (circular-shaped flow pattern) at lower Re $\cdot \lambda$ toward widespread structures that extend toward the gutters (x-shape flow pattern) at high $\mathrm{Re} \cdot \lambda$.

We attribute the significant change of the flow topology to the driving mechanisms of the flow inside the Taylor droplet. We introduce a differentiation between wall-film-based momentum transfer and the transfer across the interface of the gutters. The thickness of the wall film provides little space to accommodate a strong velocity gradient. Therefore, we consider the relative motion of the wall to be a persisting momentum source for the droplet re-circulation, which is invariant to the changes of the parameter $\operatorname{Re} \cdot \lambda$. In contrast, the motion of continuous phase through the gutters strongly depends on the flow conditions $R e$ and on the viscosity ratio $\lambda$.

Case $(\operatorname{Re} \cdot \lambda \uparrow)$ : If the liquid in the gutter is less viscous than in the droplet $(\lambda \uparrow)$, the wall-induced momentum in the gutter cannot be transferred easily to the droplet interface. The viscous droplet establishes a velocity gradient inside the gutter and possesses the $\mathrm{x}$-shape of the flow topology. At higher $\operatorname{Re}(\uparrow)$, inertia effects dominate the viscous damping of the continuous phase and the wall-film-driven mechanism is stronger than the gutter influence. Thus, the $\mathrm{x}$-shape of the topology is created.

Case $(\operatorname{Re} \cdot \lambda \downarrow)$ : If the liquid in the gutter is highly viscous compared to the droplet viscosity $(\lambda \downarrow)$, the channel wall in the gutter transfers momentum easily to the gutter interface and into the droplet. In this case, the momentum transfer via the gutter interface is as efficient as the wall-film-driven momentum transfer. The according velocity gradient is situated inside the droplet, shifts the vortex core to the droplet rear and results in the circular shape of the observed topology. At low $\operatorname{Re}(\downarrow)$, elevated viscous forces of the continuous phase result in a decreased absolute fluid mobility inside the gutter, which can be simultaneously considered to act as an increased relative fluid transport from the droplet front to its rear. The backward-directed fluid transport supports the backward motion of the wall film and establishes the observed circular shape of the topology.

The dimensionless excess velocity $u_{\mathrm{ex}}$ of a Taylor droplet is linearly related to the net volume flow that bypasses the droplet in a relative frame of reference through the gutter $\frac{Q_{g}}{Q_{\text {tot }}}$. The net flow through the gutter correlates significantly with the change of the downstream flow topology in the cross section of the main vortex of the Taylor droplet. We identify a dependence of the dimensionless friction factor of the gutter $\beta$ on the relative gutter length $\frac{l_{g}}{W}$ and the momentum coupling $\operatorname{Re} \cdot \lambda$. The stronger the momentum coupling $\operatorname{Re} \cdot \lambda$ is, the more the relative net gutter flow $\frac{Q_{\mathrm{g}}}{Q_{\text {tot }}}$ is suppressed and the excess velocity $u_{\text {ex }}$ decreases. We suggest a new correlation. However, more data are required for validation.

In order to counteract a distribution of excess velocities in a continuous Taylor droplet chain, the relative net flow through the gutter can be reduced by adjusting the following parameters of the dimensionless friction factor $\beta$ : increased gutter length $\frac{l_{\mathrm{g}}}{W}$ (long Taylor droplets), increased viscosity ratio $\lambda$ between droplet and bulk and a small Ohnesorge number $O h$ of the continuous phase, which indicates the viscous damping of inertia and surface tension forces.

\section{Outlook}

For future work, additional measurements are necessary extent in the range of dimensionless numbers $\mathrm{Ca}, \mathrm{Re}$ and $\lambda$ and their influence on the flow topology. Particularly, the effect of the secondary parameters $R e$ and $\lambda$ needs to be confirmed for a range of capillary numbers $\mathrm{Ca}$. The proposed qualitative correlation for the dimensionless friction factor of the gutter $\beta$ needs also to be validated for an extended range of dimensionless numbers $\mathrm{Ca}, \mathrm{Re}$ and $\lambda$. The accuracy of the model proposed by Helmers et al. (2019b) may be increased based on this validated quantitative correlation for the dimensionless friction factor $\beta$.

\section{Appendix}

\section{Details of the performed measurements}

The relevant dimensionless quantities, as well as the mass fractions of the flows phases for refractive index matching are shown in Table 4. 
Table 4 Measurement overview. In all measurements, $1 \mu \mathrm{moll}^{-1}$ AOT was added to the disperse phase for tracer particle stabilization. The micelle concentration of SDS in the continuous phase is $\mathrm{CMC}_{\mathrm{SDS}}=35 \mathrm{mmol}^{-1}$. The data set (VII) has been taken from Mießner et al. (2020)

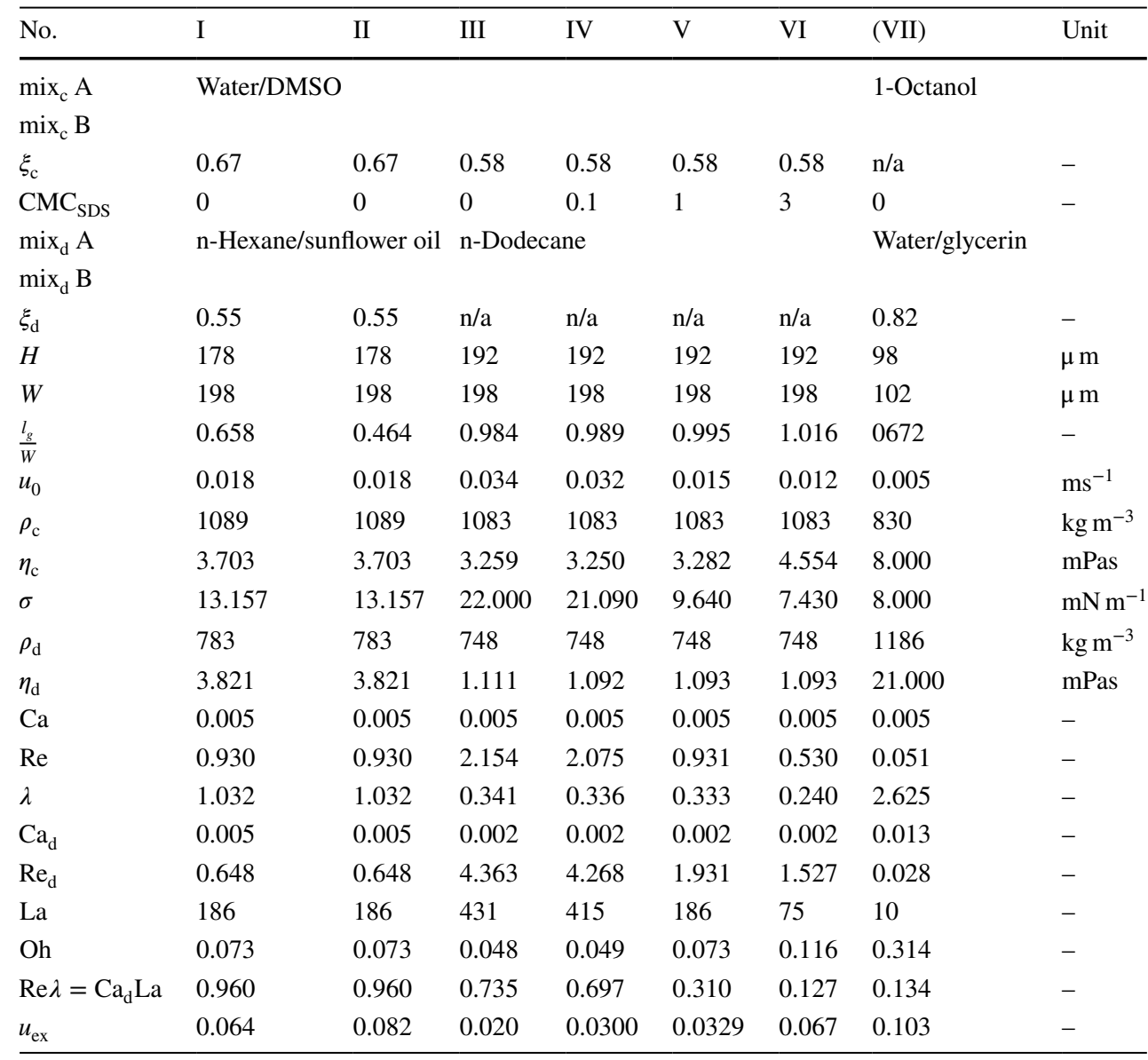

\section{Depth of correlation and adjustment of ensemble averaging}

A measurement of the downstream velocity distribution inside the gutters of Taylor droplets requires 2D flow data from multiple channels heights (multi-plane measurement approach). The $z$-distance between the measurement planes needs to exceed the depth of correlation (DOC). An overlap of measurement planes does not add information to the measurement. If the distance between two measurement planes is lower than the DOC, then information from outside the interrogated plane is included. This results in potentially biased data.

$D O F=\frac{R I \Lambda}{N A^{2}}+\frac{R I \varepsilon}{N A M}$

$\mathrm{RI}$ is the refractive index of the immersion medium between objective and substrate, $\Lambda$ the light wavelength of the fluorescence signal, $\varepsilon$ the minimal detectable area and NA the numerical aperture:

$N A=R I \sin (\theta)$
Here, $\theta$ represents the opening angle of the objective. Often the numerical aperture $N A$ is given for air. For the case of immersion objectives, $N A$ can be corrected if the RI of the immersion medium is known.

To calculate the DOC in the following, at first the numerical aperture of the objective Carl Zeiss LD LCI PlanApochromat $25 x / 0.8$ is converted to the used water with a $\mathrm{RI}=1.333$ since instead of air $(\mathrm{RI}=1)$. A half opening angle for air as immersion medium can be calculated as $\theta=53.13^{\circ}$. From this, the numeric aperture for water can be calculated as $N A=1.0666$. This results in a depth of field $D O F=1.034 \mu \mathrm{m}$, and a corrected $D O C_{\text {corr }}=7.034 \mu \mathrm{m}$ is retrieved.

To prove the calculations, an image series of stagnant fluorescence particles dispersed in water/DMSO is acquired. Fluorescence particles are dispersed in water/DMSO, placed on a microscope slide, and are embedded by a coverslip. The particles are illuminated by a laser, and the fluorescence signal is recorded. The $z$-position of the objective is adjusted for the sharpest image of the interrogated particle and subsequently varied $10 \mu \mathrm{m}$ around the focal point, while for every $z$-step an image is acquired (Fig. 15).

Both image series show a rapid decrease in fluorescence intensity, and therefore loss of information increases beyond 


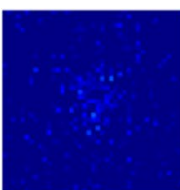

(a)

$\mathrm{z}=-4.0 \mu \mathrm{m}$

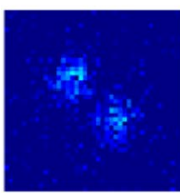

(j)

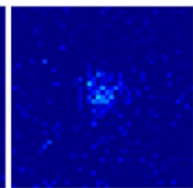

(b)

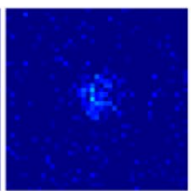

(c)

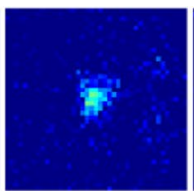

(d)

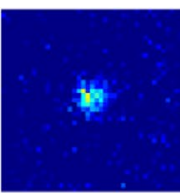

(e)

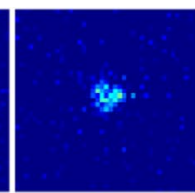

(f)

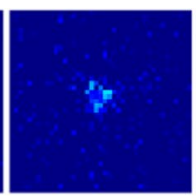

(g)

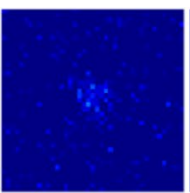

(h) $\mathrm{z}=2.0 \mathrm{\mu m}$

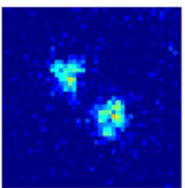

(p)

$\mathrm{z}=3.0 \mu \mathrm{m}$

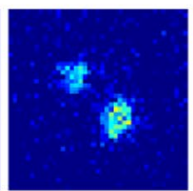

(q)

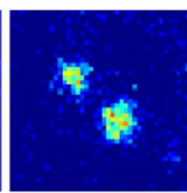

(n)

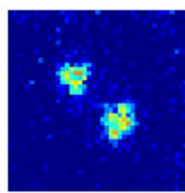

(o) (k) (l) (m)

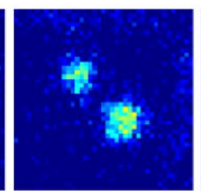

$\mathrm{z}=-4.0 \mu \mathrm{m} \quad \mathrm{z}=-3.0 \mu \mathrm{m} \quad \mathrm{z}=-2.0 \mu \mathrm{m} \quad \mathrm{z}=-1.0 \mu \mathrm{m} \quad \mathrm{z}=0 \mu \mathrm{m} \quad \mathrm{z}=1.0 \mu \mathrm{m} \quad \mathrm{z}=2.0 \mu \mathrm{m} \quad \mathrm{z}=3.0 \mu \mathrm{m} \quad \mathrm{z}=4.0 \mu \mathrm{m}$

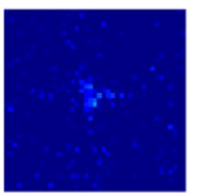

(i)

$\mathrm{z}=4.0 \mu \mathrm{m}$

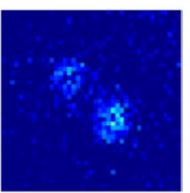

(r)

Fig. 15 Validation of calculated depth of correlation using two images series ( $\mathbf{a}-\mathbf{i}$ and $\mathbf{j}-\mathbf{r}$ ) of $1.61 \mu \mathrm{m}$ fluorescence particles at different z-positions deviating from the particle (pixel intensity is coded as color). Each single image represents an area of $7 \mu \mathrm{m} \cdot 7 \mu \mathrm{m}$

a $z$-position of $3 \mu \mathrm{m}$ to $4 \mu \mathrm{m}$ from the focal point. Thus, judging from the presented image series, a $D O C_{\text {meas }}$ of $6 \mu \mathrm{m}$ to $8 \mu \mathrm{m}$ is retrieved. This is in accordance with the calculated value $D O C_{\text {corr }}=7 \mu \mathrm{m}$. Therefore, a mean distance of at least $7 \mu \mathrm{m}$ is chosen between the $z$-positions of the measurement planes.

\section{Post-processing of the droplet raw images}

The post-processing of the droplet raw images is performed using an ensemble image averaging approach. For the sorting of the raw images, the intensity-based sorting and shifting approach by Mießner et al. (2019) is used. As an improvement in this work, the tolerable droplet length deviation over all measurement planes, as well as the used median droplet length, is acquired via an iterative approximation approach: The considered median droplet length is chosen such that for all 14 measurement planes at least 30 droplets are available for post-processing. If this number is exceeded, the tolerable droplet length deviation is subsequently decreased until only the desired 30 droplets are remaining. This is performed for several median droplet lengths and finally the median length with the least droplet length deviation over all planes is used.

\section{Measurement of Mießner et al. (2020)}

Open access measurement data are published by Mießner et al. (2020) $(C a=0.005, R e=0.051, \lambda=2.625$, $\left.u_{e x}=0.1028\right)$. The core of the main vortex is shifted toward the rear of the droplet, despite the clearly elevated viscosity ration between the aqueous droplet phase (water-glycerol) and the nonpolar continuous phase (1-octanol). The outer flow does not provide sufficient momentum to counteract the reversed bypass flow through the gutters toward the

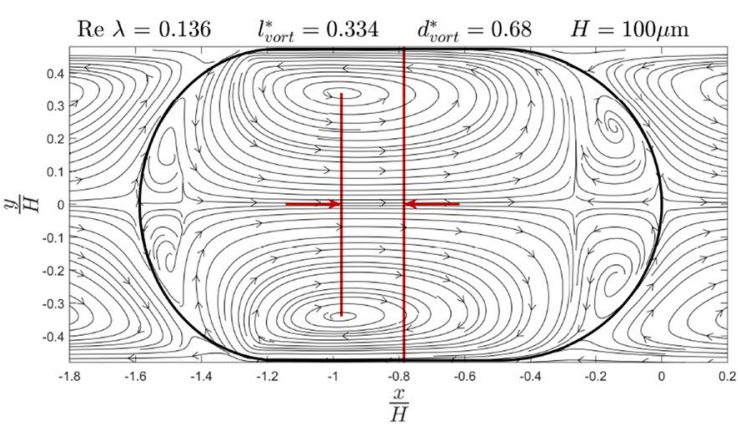

Fig. 16 Measurement of Mießner et al. (2020). Left: Streamline plot of the relative velocity field in the symmetry plane of the microchannel. The geometrical droplet center and the center position of the main vortex ring are marked with a red line. Center: Distribution of the downstream relative velocities. The flow topology exhibits a circular shape, despite the elevated viscosity ratio $\lambda$. However, iner-
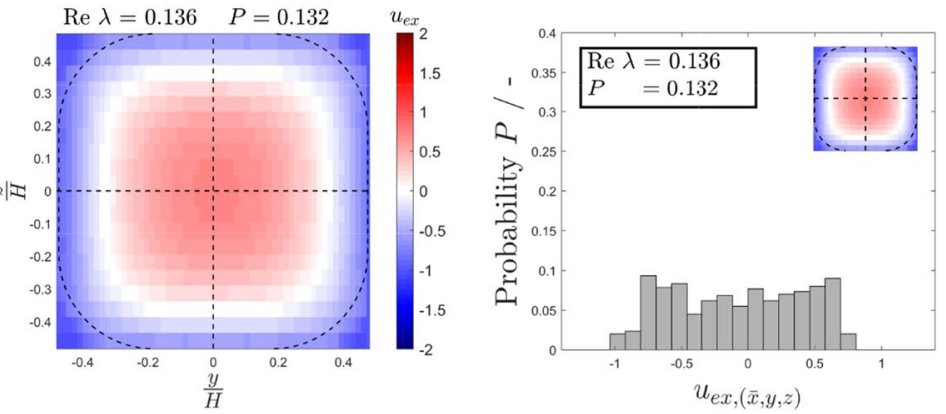

tia forces are dominated by the viscous forces, such that the $\mathrm{x}$-shape topology cannot be established. Right: Probability distribution of the downstream relative velocity distribution. The combined probability of the bins, which represents the stagnating fractions $u_{\mathrm{ex}} \approx 0$, amounts to $P=0.136$. The measure $P$ is used in the context of this study to quantify the topological state of the flow 


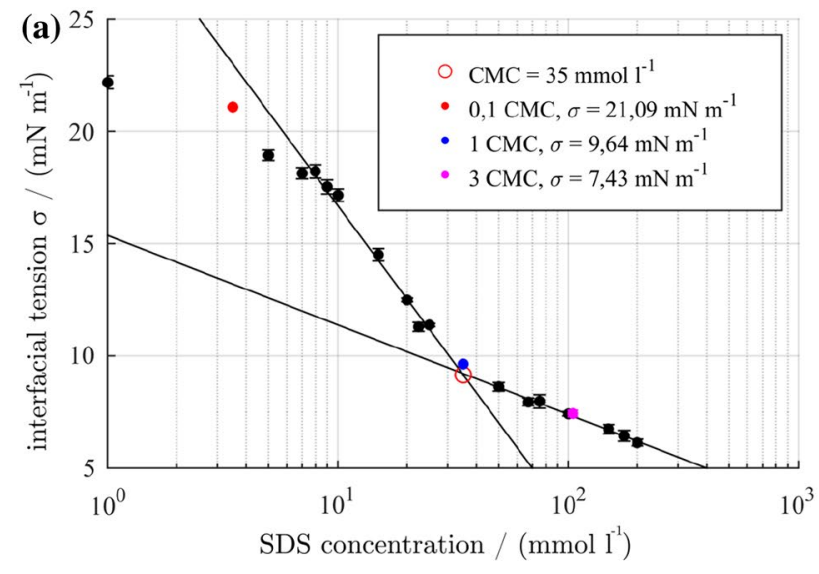

(b)

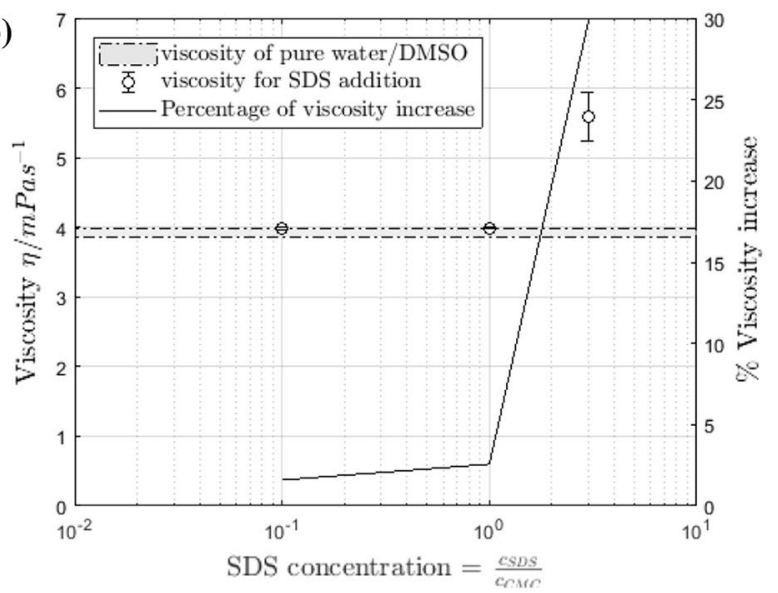

Fig. 17 a Measurements of the interfacial tension for different SDS concentrations in a mixture of water/DMSO with mass-fraction $\xi=0.58$ and $1 \mu \mathrm{mol} l^{-1}$ AOT in the n-dodecane fraction $\mathbf{b}$ Viscosity measurements for water/DMSO $\xi=0.58$ with addition of SDS. Measurements were performed using a Bohlin rheometer CS in a double-gap configuration at $\mathrm{T}=21^{\circ} \mathrm{C}$

droplet rear. As a consequence, the velocity gradient is situated inside the droplet and a circularly shaped topology is established (Fig. 16).

\section{Measurements for RIM and influence of surfactants on the flow}

Interfacial tension measurements are performed to measure the water/DMSO mixture with $1 \mu \mathrm{moll}^{-1}$ AOT added in combination with n-dodecane. A variety of SDS concentrations is dissolved in water/DMSO $(\xi=0.58)$, and the interfacial tension is measured. The critical micelle concentration $\left(\mathrm{CMC}=35 \mathrm{mmol}^{-1}\right.$, Table, 4$)$ for SDS is retrieved from the point, where the slope $\frac{d \sigma}{d c_{\mathrm{SDS}}}$ changes.

The addition of SDS to the mixture of water/glycerol leads to an increase in the dynamic viscosity of the mixture.

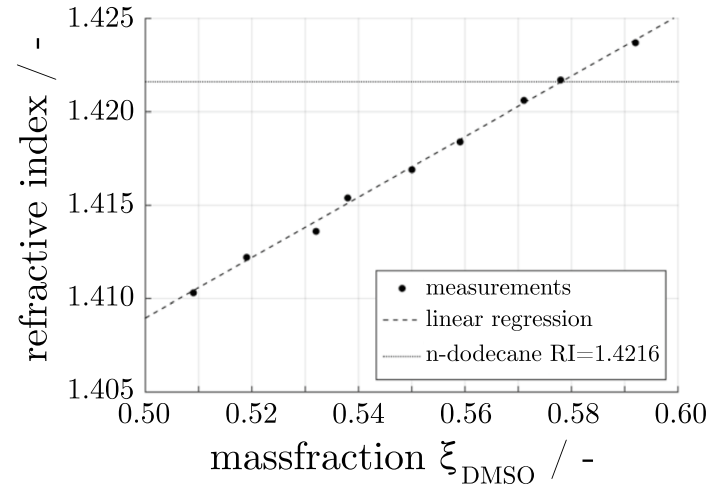

Fig. 18 Measurements of refractive index for water/DMSO mixture at different mass fractions $\xi$. At $\xi=0.58$, the refractive index of the mixture equals the RI of $\mathrm{n}$-dodecane

Below the CMC, the viscosity increase is moderate, while for concentration over the CMC the viscosity is increased by up to $30 \%$ (Fig. 17b).

The necessary mass fraction of the water/DMSO flow phase is retrieved from refractive index measurements (Fig 18).

Acknowledgements The contribution of P. Kemper has been supported by the German Research Foundation (DFG), Priority Program: "Reactive Bubbly Flows," SPP1740 under grant TH 893/17-2. The authors gratefully acknowledge the financial support.

Author contributions TH and PK contributed equally; TH, PK, JT and UM contributed to conceptualization and formal analysis; TH and UM helped in methodology; TH, PK and UM contributed to software and validation; TH and PK performed investigation, writing - original draft preparation, and visualization; UM and JT contributed to resources, writing - review and editing, and supervision; UM curated the data.

Funding Open Access funding enabled and organized by Projekt DEAL.

Open Access This article is licensed under a Creative Commons Attribution 4.0 International License, which permits use, sharing, adaptation, distribution and reproduction in any medium or format, as long as you give appropriate credit to the original author(s) and the source, provide a link to the Creative Commons licence, and indicate if changes were made. The images or other third party material in this article are included in the article's Creative Commons licence, unless indicated otherwise in a credit line to the material. If material is not included in the article's Creative Commons licence and your intended use is not permitted by statutory regulation or exceeds the permitted use, you will need to obtain permission directly from the copyright holder. To view a copy of this licence, visit http://creativecommons.org/licenses/by/4.0/.

\section{References}

Adrian R, Westerweel J (2011) Particle image velocimetry, Cambridge aerospace series, vol 30. Cambridge Univ. Press, Cambridge 
Angeli P, Gavriilidis A (2008) Hydrodynamics of taylor flow in small channels: a review. Proc Ins Mech Eng Part C J Mech Eng Sci 222(5):737-751. https://doi.org/10.1243/09544062JMES776

Budwig R (1994) Refractive index matching methods for liquid flow investigations. Exp Fluids 17(5):350-355. https://doi.org/10.1007/ BF01874416

Chambers RD, Holling D, Rees AJ, Sandford G (2003) Microreactors for oxidations using fluorine. ChemInform. https://doi.org/ 10.1002/chin.200317049

Chen J, Song W, Xu D (2018) Catalytic partial oxidation of methane for the production of syngas using microreaction technology: a computational fluid dynamics study. Int J Hydrog Energy 43(31):14059-14077. https://doi.org/10.1016/j.ijhydene.2018.05. 125

Chou WL, Lee PY, Yang CL, Huang WY, Lin YS (2015) Recent advances in applications of droplet microfluidics. Micromachines 6(9):1249-1271. https://doi.org/10.3390/mi6091249

Delnoij E, Westerweel J, Deen N, Kuipers J, van Swaaij W (1999) Ensemble correlation piv applied to bubble plumes rising in a bubble column. Chem Eng Sci 54(21):5159-5171. https://doi.org/ 10.1016/S0009-2509(99)00233-X

Dietrich TR (2009) Microchemical engineering in practice. Wiley, Hoboken, NJ and Hoboken, NJ. https://doi.org/10.1002/97804 70431870

Ehrfeld W, Hessel V, Lowe H (2004) Microreactors: new technology for modern chemistry. Wiley-VCH, Weinheim. https://doi.org/10. $1002 / 3527601953$

Ern P, Risso F, Fabre D, Magnaudet J (2012) Wake-induced oscillatory paths of bodies freely rising or falling in fluids. Ann Rev Fluid Mech 44(1):97-121. https://doi.org/10.1146/annur ev-fluid-120710-101250

Fuerstman MJ, Lai A, Thurlow ME, Shevkoplyas SS, Stone HA, Whitesides GM (2007) The pressure drop along rectangular microchannels containing bubbles. Lab Chip 7(11):1479-1489. https:// doi.org/10.1039/b706549c

Haase S, Murzin DY, Salmi T (2016) Review on hydrodynamics and mass transfer in minichannel wall reactors with gas-liquid taylor flow. Chem Eng Res Des 113:304-329. https://doi.org/ 10.1016/j.cherd.2016.06.017

Han Y, Shikazono N (2009) Measurement of the liquid film thickness in micro tube slug flow. Int J Heat Fluid Flow 30(5): 842 853. https://doi.org/10.1016/j.ijheatfluidflow.2009.02.019. The 3rd international conference on heat transfer and fluid flow in microscale

Helmers T, Kemper P, Thöming J, Mießner U (2019) Determining the flow-related cap deformation of taylor droplets at low ca numbers using ensemble-averaged high-speed images. Exp Fluids 60(7):66. https://doi.org/10.1007/s00348-019-2757-7

Helmers T, Kemper P, Thöming J, Mießner U (2019) Modeling the excess velocity of low-viscous taylor droplets in square microchannels. Fluids 4(3):162. https://doi.org/10.3390/fluids4030 162

Helmers T, Kemper P, Mießner U, Thöming J (2020) Refractive index matching (rim) using double-binary liquid-liquid mixtures. Exp Fluids 61(2):64. https://doi.org/10.1007/s00348-020-2892-1

Huebner A, Sharma S, Srisa-Art M, Hollfelder F, Edel JB, Demello AJ (2008) Microdroplets: a sea of applications? Lab Chip 8(8):1244 1254. https://doi.org/10.1039/b806405a

Jakiela S, Makulska S, Korczyk PM, Garstecki P (2011) Speed of flow of individual droplets in microfluidic channels as a function of the capillary number, volume of droplets and contrast of viscosities. Lab Chip 11(21):3603-3608. https://doi.org/10.1039/c1lc20534j

Jakiela S, Korczyk PM, Makulska S, Cybulski O, Garstecki P (2012) Discontinuous transition in a laminar fluid flow: a change of flow topology inside a droplet moving in a micron-size channel. Phys
Rev Lett 108(13):134501. https://doi.org/10.1103/PhysRevLett. 108.134501

Jose BM, Cubaud T (2014) Formation and dynamics of partially wetting droplets in square microchannels. RSC Adv 4:14962-14970. https://doi.org/10.1039/C4RA00654B

Kinoshita H, Kaneda S, Fujii T, Oshima M (2007) Three-dimensional measurement and visualization of internal flow of a moving droplet using confocal micro-piv. Lab Chip 7:338-346. https://doi.org/ 10.1039/B617391H

Kobayashi J, Mori Y, Kobayashi S (2006) Multiphase organic synthesis in microchannel reactors. Chem Asian J 1(1-2):22-35. https://doi. org/10.1002/asia.200600058

Kovalchuk NM, Simmons MJH (2021) Effect of surfactant dynamics on flow patterns inside drops moving in rectangular microfluidic channels. Colloids Interfaces. https://doi.org/10.3390/colloids50 30040

Kovalev AV, Yagodnitsyna AA, Bilsky AV (2018) Flow hydrodynamics of immiscible liquids with low viscosity ratio in a rectangular microchannel with t-junction. Chem Eng J 352:120-132. https:// doi.org/10.1016/j.cej.2018.07.013

Kralj JG, Sahoo HR, Jensen KF (2007) Integrated continuous microfluidic liquid-liquid extraction. Lab Chip 7(2):256-263. https:// doi.org/10.1039/b610888a

Kreutzer M, Kapteijn F, Moulijn J, Heiszwolf J (2005) Multiphase monolith reactors: chemical reaction engineering of segmented flow in microchannels. Chem Eng Sci 60(22):5895-5916. https:// doi.org/10.1016/j.ces.2005.03.022. 7th international conference on gas-liquid and gas-liquid-solid reactor engineering

Lang P, Hill M, Krossing I, Woias P (2012) Multiphase minireactor system for direct fluorination of ethylene carbonate. Chem Eng J 179:330-337. https://doi.org/10.1016/j.cej.2011.11.015

Leong CM, Gai Y, Tang SKY (2016) Internal flow in droplets within a concentrated emulsion flowing in a microchannel. Phys Fluids 28(11):112001. https://doi.org/10.1063/1.4968526

Li M, Liu Z, Pang Y, Yan C, Wang J, Zhao S, Zhou Q (2020) Flow topology and its transformation inside droplets traveling in rectangular microchannels. Phys Fluids 32(5):052009. https://doi. org/10.1063/5.0004549

Lindken R, Rossi M, Große S, Westerweel J (2009) Micro-particle image velocimetry ( $\mu$ piv): recent developments, applications, and guidelines. Lab Chip 9:2551-2567. https://doi.org/10.1039/ B906558J

Liu Z, Zhang L, Pang Y, Wang X, Li M (2017) Micro-piv investigation of the internal flow transitions inside droplets traveling in a rectangular microchannel. Microfluid Nanofluidics 21(12):180. https://doi.org/10.1007/s10404-017-2019-z

Luo ZY, Shang XL, Bai BF (2019) Effect of soluble surfactant on the motion of a confined droplet in a square microchannel. Phys Fluids 31(11): 117104. https://doi.org/10.1063/1.5125949

Ma S, Sherwood JM, Huck WTS, Balabani S (2014) On the flow topology inside droplets moving in rectangular microchannels. Lab Chip 14:3611-3620. https://doi.org/10.1039/C4LC00671B

Magnaudet J, Eames I (2000) The motion of high-reynolds-number bubbles in inhomogeneous flows. Annu Rev Fluid Mech 32(1):659-708. https://doi.org/10.1146/annurev.fluid.32.1.659

Makeev IV, Popov IY, Abiev RS (2019) Analytical solution of taylor circulation in a prolate ellipsoid droplet in the frame of $2 \mathrm{~d}$ stokes equations. Chem Eng Sci 207:145-152. https://doi.org/10.1016/j. ces.2019.06.015

Mießner U, Helmers T, Lindken R, Westerweel J (2019) An analytical interface shape approximation of microscopic taylor flows. Exp Fluids 60(4):75. https://doi.org/10.1007/s00348-019-2719-0

Mießner U, Helmers T, Lindken R, Westerweel J (2020) $\mu$ piv measurement of the $3 \mathrm{~d}$ velocity distribution of taylor droplets moving in a square horizontal channel. Exp Fluids 61(5):125. https://doi.org/ 10.1007/s00348-020-02949-z 
Mießner U, Lindken R, Westerweel J (2008) Velocity measurements in microscopic two-phase flows by means of micro piv. In: Proceedings of the 6th international conference on nanochannels, microchannels and minichannels-2008. ASME, New York, NY, pp 1111-1118. https://doi.org/10.1115/ICNMM2008-62093

Oishi M, Kinoshita H, Fujii T, Oshima M (2011) Simultaneous measurement of internal and surrounding flows of a moving droplet using multicolour confocal micro-particle image velocimetry micro-piv. Meas Sci Technol 22(10):105401. https://doi.org/10. 1088/0957-0233/22/10/105401

Olgac U, Muradoglu M (2013) Effects of surfactant on liquid film thickness in the bretherton problem. Int J Multiph Flow 48:58-70. https://doi.org/10.1016/j.ijmultiphaseflow.2012.08.007

Olsen MG (2008) Directional dependence of depth of correlation due to in-plane fluid shear in microscopic particle image velocimetry. Meas Sci Technol 20(1):105402. https://doi.org/10.1088/09570233/20/1/015402

Olsen MG (2010) Depth of correlation reduction due to out-of-plane shear in microscopic particle image velocimetry. Meas Sci Technol 21(10):105406. https://doi.org/10.1088/0957-0233/21/10/ 105406

Olsen MG, Adrian RJ (2000) Out-of-focus effects on particle image visibility and correlation in microscopic particle image velocimetry. Exp Fluids 29(1):S166-S174. https://doi.org/10.1007/s0034 80070018

Raffel M, Willert CE, Scarano F, Kähler CJ, Wereley ST, Kompenhans J (2018) Particle image velocimetry: a practical guide, 3rd edn. Springer International Publishing, Cham, UK. https://doi.org/10. 1007/978-3-319-68852-7

Ransohoff T, Radke C (1988) Laminar flow of a wetting liquid along the corners of a predominantly gas-occupied noncircular pore. J Colloid Interface Sci 121(2):392-401. https://doi.org/10.1016/ 0021-9797(88)90442-0

Rao SS, Wong H (2018) The motion of long drops in rectangular microchannels at low capillary numbers. J Fluid Mech 852:60104. https://doi.org/10.1017/jfm.2018.521

Rossetti I (2018) Continuous flow (micro-)reactors for heterogeneously catalyzed reactions: main design and modelling issues. Catal Today 308:20-31. https://doi.org/10.1016/j.cattod.2017.09.040

Rossi M, Lindken R, Westerweel J (2010) Optimization of multiplane $\mu$ piv for wall shear stress and wall topography characterization. Exp Fluids 48(2):211-223. https://doi.org/10.1007/s00348-009-0725-3

Sattari-Najafabadi M, Nasr Esfahany M, Wu Z, Sunden B (2018) Mass transfer between phases in microchannels: a review. Chem Eng Process Process Intensif 127:213-237. https://doi.org/10.1016/j. cep.2018.03.012

Sauzade M, Cubaud T (2013) Initial microfluidic dissolution regime of $\mathrm{co} 2$ bubbles in viscous oils. Phys Rev E Stat Nonlinear Soft Matter Phys 88(5):051,001. https://doi.org/10.1103/PhysRevE. 88.051001
Seeberger PH, Blume T (2007) New avenues to efficient chemical synthesis: emerging technologies. In: Ernst schering foundation symposium proceedings, vol 2006/3. Springer-Verlag, Berlin Heidelberg, Berlin, Heidelberg. https://doi.org/10.1007/ 978-3-540-70849-0

Shams M, Raeini AQ, Blunt MJ, Bijeljic B (2018) A study to investigate viscous coupling effects on the hydraulic conductance of fluid layers in two-phase flow at the pore level. J Colloid Interface Sci 522:299-310. https://doi.org/10.1016/j.jcis.2018.03.028

Shi H, Nie K, Dong B, Long M, Xu H, Liu Z (2019) Recent progress of microfluidic reactors for biomedical applications. Chem Eng J 361:635-650. https://doi.org/10.1016/j.cej.2018.12.104

Song H, Chen DL, Ismagilov RF (2006) Reactions in droplets in microfluidic channels. Angew Chem (International ed in English) 45(44):7336-7356. https://doi.org/10.1002/anie.200601554

Sun B, Jiang J, Shi N, Xu W (2016) Application of microfluidics technology in chemical engineering for enhanced safety. Process Saf Prog 35(4):365-373. https://doi.org/10.1002/prs.11801

Tanimu A, Jaenicke S, Alhooshani K (2017) Heterogeneous catalysis in continuous flow microreactors: a review of methods and applications. Chem Eng J 327:792-821. https://doi.org/10.1016/j.cej. 2017.06.161

van Steijn V, Kleijn CR, Kreutzer MT (2009) Flows around confined bubbles and their importance in triggering pinch-off. Phys Rev Lett 103(214):501. https://doi.org/10.1103/PhysRevLett.103. 214501

Vennemann P, Kiger KT, Lindken R, Groenendijk BC, de Vos SS, ten Hagen TL, Ursem NT, Poelmann RE, Westerweel J, Hierck BP (2006) In vivo micro particle image velocimetry measurements of blood-plasma in the embryonic avian heart. J Biomech 39(7):1191-1200. https://doi.org/10.1016/j.jbiomech.2005.03.015

Wong S, Ward M, Wharton C (2004) Micro t-mixer as a rapid mixing micromixer. Sens Actuators B Chem 100(3):359-379. https://doi. org/10.1016/j.snb.2004.02.008

Wright SF, Zadrazil I, Markides CN (2017) A review of solid-fluid selection options for optical-based measurements in single-phase liquid, two-phase liquid-liquid and multiphase solid-liquid flows. Exp Fluids 58(9):357. https://doi.org/10.1007/s00348-017-2386-y

Yusuf A, Garlisi C, Palmisano G (2018) Overview on microfluidic reactors in photocatalysis: applications of graphene derivatives. Catal Today 315:79-92. https://doi.org/10.1016/j.cattod.2018.05. 041

Zhao CX, Middelberg AP (2011) Two-phase microfluidic flows. Chem Eng Sci 66(7):1394-1411. https://doi.org/10.1016/j.ces.2010.08. 038

Publisher's Note Springer Nature remains neutral with regard to jurisdictional claims in published maps and institutional affiliations.

\section{Authors and Affiliations}

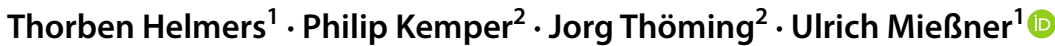

Thorben Helmers

helmers@uvt.uni-bremen.de

Philip Kemper

pkemper@uni-bremen.de

Jorg Thöming

thoeming@uni-bremen.de
1 Research Group Environmental Process Engineering, University of Bremen, Leobener Str. 6, 28359 Bremen, Germany

2 Department of Chemical Process Engineering, University of Bremen, Leobener Str. 6, 28359 Bremen, Germany 